Research paper

\title{
A major role of TWEAK/Fn14 axis as a therapeutic target for post-angioplasty restenosis
}

\author{
Nerea Méndez-Barbero ${ }^{\mathrm{a}, 1}$, Carmen Gutierrez-Muñoz ${ }^{\mathrm{a}, 1}$, Julio Madrigal-Matute ${ }^{\mathrm{b}}$, Pablo Mínguez ${ }^{\mathrm{c}}$, Jesús Egido ${ }^{\mathrm{d}}$, \\ Jean-Baptiste Michel ${ }^{\mathrm{e}}$, Jose L. Martín-Ventura ${ }^{\mathrm{a}}$, Vanesa Esteban ${ }^{\mathrm{f}, *, 2}$, Luis M. Blanco-Colio ${ }^{\mathrm{a}, *, 2}$ \\ ${ }^{a}$ Vascular Research Lab, CIBERCV, IIS-Fundación Jiménez Díaz, Madrid, Spain \\ ${ }^{\mathrm{b}}$ Department of Developmental and Molecular Biology, Albert Einstein College of Medicine, New York, USA \\ c Department of Genetics and Genomics, IIS-Fundación Jiménez Díaz, Madrid, Spain \\ d Renal and Diabetes Research Lab, CIBERDEM, IIS-Fundación Jiménez Díaz, Madrid, Spain \\ e INSERM U1148, Laboratory for Vascular Translational Science (LVTS), Paris, France \\ ${ }^{\mathrm{f}}$ Department of Immunology and ARADyAL, IIS-Fundación Jiménez Díaz, Madrid, Spain
}

\section{A R T I C L E I N F O}

\section{Article history:}

Received 12 February 2019

Received in revised form 30 July 2019

Accepted 30 July 2019

Available online 5 August 2019

\section{Keywords:}

Restenosis

Proliferation

Cyclins

TWEAK

Fn14

\begin{abstract}
A B S T R A C T
Background: Tumor necrosis factor-like weak inducer of apoptosis (Tnfsf12; TWEAK) and its receptor Fibroblast growth factor-inducible 14 (Tnfrsf12a; Fn14) participate in the inflammatory response associated with vascular remodeling. However, the functional effect of TWEAK on vascular smooth muscle cells (VSMCs) is not completely elucidated.

Methods: Next generation sequencing-based methods were performed to identify genes and pathways regulated by TWEAK in VSMCs. Flow-citometry, wound-healing scratch experiments and transwell migration assays were used to analyze VSMCs proliferation and migration. Mouse wire injury model was done to evaluate the role of TWEAK/Fn14 during neointimal hyperplasia.

Findings: TWEAK up-regulated 1611 and down-regulated 1091 genes in VSMCs. Using a gene-set enrichment method, we found a functional module involved in cell proliferation defined as the minimal network connecting top TWEAK up-regulated genes. In vitro experiments in wild-type or Tnfrsf12a deficient VSMCs demonstrated that TWEAK increased cell proliferation, VSMCs motility and migration. Mechanistically, TWEAK increased cyclins (cyclinD1), cyclin-dependent kinases (CDK4, CDK6) and decreased cyclin-dependent kinase inhibitors $\left(\mathrm{p} 15^{\mathrm{INK} 4 \mathrm{~B}}\right)$ mRNA and protein expression. Downregulation of $\mathrm{p} 15^{\mathrm{INK} 4 \mathrm{~B}}$ induced by TWEAK was mediated by mitogen-activated protein kinase ERK and Akt activation.

Tnfrsf12a or Tnfsf12 genetic depletion and pharmacological intervention with TWEAK blocking antibody reduced neointimal formation, decreasing cell proliferation, cyclin D1 and CDK4/6 expression, and increasing p15 ${ }^{\mathrm{INK} 4 \mathrm{~B}}$ expression compared with wild type or IgG-treated mice in wire-injured femoral arteries. Finally, immunohistochemistry in human coronary arteries with stenosis or in-stent restenosis revealed high levels of Fn14, TWEAK and PCNA in VSMCs enriched areas of the neointima as compared with healthy coronary arteries.

Interpretation: Our data define a major role of TWEAK/Fn14 in the control of VSMCs proliferation and migration during neointimal hyperplasia after wire injury in mice, and identify TWEAK/Fn14 as a potential target for treating in-stent restenosis.

Fund: ISCiii-FEDER, CIBERCV and CIBERDEM.
\end{abstract}

(C) 2019 Published by Elsevier B.V. This is an open access article under the CC BY-NC-ND license (http:// creativecommons.org/licenses/by-nc-nd/4.0/).

\section{Introduction}

Pathological vascular wall remodeling is a critical feature of vascular diseases such as atherosclerosis, post-angioplasty restenosis, vein graft

\footnotetext{
* Corresponding authors.

E-mail addresses: vesteban@fjd.es (V. Esteban), lblanco@fjd.es (L.M. Blanco-Colio).

1 Both authors contributed equally to this work.

2 Equal contribution of the last 2 senior authors.
}

stenosis, abdominal aortic aneurysm, and vasculopathy after transplantation [1]. Vascular smooth muscle cells (VSMCs) are key players in adult vascular remodeling due to their remarkable phenotypic plasticity [2]. Under physiological conditions, VSMCs are quiescent, contractile and non-migratory in the vessel wall. However, in response to vascular injury the resident medial VSMCs are activated proliferating and migrating into the intima, where they accumulate and subsequently produce proinflammatory cytokines and chemokines and abundant 
extracellular matrix proteins to form the neointima [3,4]. Intimal hyperplasia formation and restenosis can be controlled by the use of drugeluting stents. However, the in-stent restenosis still occur in $10 \%$ patients and remained a significant clinical problem to be solved [5].

Several studies have shed light on some of the pathophysiological mechanisms that are involved in VSMCs proliferation and migration. Nevertheless the identification of molecular mediators that link these coordinated responses of VSMCs to injury could help to design new and selective treatment strategies to prevent VSMCs activation [6].

Tumor necrosis factor-like weak inducer of apoptosis (TWEAK, Tnfsf12) and its cognate receptor fibroblast growth factor-inducible 14 (Fn14, Tnfrsf12a) belong to the tumor necrosis factor superfamily of proteins [7]. Several physiological and pathological processes are induced by TWEAK depending of the cell type and environment [8]. In the vasculature, TWEAK is expressed in both the healthy and the pathological arterial wall [9]. Whereas Fn14 is low or absent in normal arteries, it is highly abundant under pathological conditions including atherosclerosis and abdominal aortic aneurysm [9-11]. Several stimuli such as endothelial growth factor, Interferon- $\gamma$, Interleukin-1 $\beta$, thrombin and angiotensin stimulate Fn14 expression in cultured VSMCs [7,9]. During pathological vascular wall remodeling, TWEAK/Fn14 axis participates in regulating endothelial dysfunction, inflammation, angiogenesis and thrombosis [12]. Thus, TWEAK injection aggravates atherosclerotic plaque development in hyperlipidemic apolipoprotein-E deficient mice by boosting the inflammatory response, among other detrimental processes [13]. Conversely, lack of $T n f s f 12$ gene or in vivo neutralizing using Fn14 and TWEAK antibodies reduces atherosclerotic lesion size and increases plaque stability $[11,13,14]$. In addition, loss-of function approaches have demonstrated that TWEAK/Fn14 axis participates in the development of abdominal aortic aneurysms in mice through the regulation of the proinflammatory and metalloproteinase environment [10].

Thus, the TWEAK/Fn14 axis exerts a key regulatory role during vascular remodeling, nonetheless the TWEAK-Fn14 downstream mechanisms are yet to be defined. Therefore, we performed a genome-wide RNA sequencing screen in cultured VSMCs aiming to identify the molecular mediators of the TWEAK/Fn14 axis. The in-silico analysis of the RNA-seq data using network biology software demonstrates that one of the main biological processes modulated by TWEAK is associated to cell proliferation. In line with this analysis, we mechanistically demonstrate that TWEAK in VSMCs increases cyclin D1 and CDK4/6 and decreases $\mathrm{p} 15^{\mathrm{INK} 4 \mathrm{~B}}$ expression, which in turn leads to activate VSMCs proliferation and migration. In addition, we validated our results in vivo since we show in this work that TWEAK accelerates neointimal formation after wire injury in femoral arteries. Finally, we demonstrate that pharmacological intervention with anti-TWEAK antibody reduced neointimal hyperplasia after wire injury in mice. These findings indicate that the TWEAK/Fn14 system may represent an attractive potential therapeutic target for treating vascular remodeling, including restenosis after angioplasty.

\section{Materials and methods}

\subsection{Cell culture}

Aortic VSMCs were isolated from aorta of wild-type (WT) and Tnfrsf12a deficient mice [15]. Briefly, adhering fat and connective tissue were removed by blunt dissection from the thoracic aorta. Aortas were minced into small ring and preincubated in DMEM (Whitaker) containing $1 \mathrm{mg} / \mathrm{mL}$ collagenase (type II, $290 \mathrm{U} / \mathrm{mg}$ ), penicillin $(100 \mathrm{U} / \mathrm{mL})$, streptomycin $(100 \mathrm{lg} / \mathrm{mL})$, and glutamine $(2 \mathrm{mmol} / \mathrm{L}$ ) (Sigma) for 15 to $20 \mathrm{~min}$ at $37^{\circ} \mathrm{C}$ in $95 \%$ air $/ 5 \% \mathrm{CO}_{2}$ and then explants were seeded in DMEM with 10\% FBS. Cells were harvested for passaging at 2- to 3-day intervals and used between the second and seventh passages. VSMCs were stained with specific markers of VSMC $\alpha$-SMA (A2547; Sigma) and calponin (ab46794; Abcam), endothelial cells (CD31, ab28364; Abcam) and fibroblast (S100A4, ab93283; Abcam) to ensure the purity of VSMC isolation methods (Fig. S1). For experimental analysis, cells were made quiescent by 24 -h incubation in medium with $0-0.5 \%$ FBS.

\subsection{RNA-Seq library construction and sequencing}

RNA-Seq libraries were prepared using the Illumina TruSeq Stranded Total RNA library prep, after ribodepletion with the Epicenter Ribozero Gold kit (cat\# RZE1224) starting from 500 ng of DNAse I treated total RNA, following the manufacturer's protocol, with the exception that 14 cycles of PCR were performed to amplify the libraries, to keep the duplication rate lower than with the recommended 15 cycles. The amplified libraries were purified using AMPure beads, quantified by Qubit and QPCR, and visualized in an Agilent Bioanalyzer. The libraries were pooled equimolarly, and loaded on an Illumina HiSeq 2500 flow cell, $\mathrm{v} 4$ chemistry as paired end 50 . The R statistical software environment was used to run the Bioconductor package, DESeq2 to analyze the RNA-Seq data set for differential expression between groups (Applied Bioinformatics Laboratory, NYU School of Medicine, New York, USA).

\subsection{Gene set enrichment}

A method for gene set enrichment analysis based on logistic regression [16] implemented in the Babelomics suite [17] was used to extract the GO terms (biological processes, molecular functions and cellular components) and KEGG pathways (annotation extracted using KEGG rest service) over-represented in each of the conditions. A threshold of adjusted $p$-value by FDR $<0.05$ was used to select the significant GO terms and KEGG pathways.

\subsection{Functional module extraction and functional enrichment}

The NetworkMiner [18] web-tool implemented in the Babelomics suite [17] was used to extract the relevant protein-protein interaction networks associated to each of the two conditions compared. The input for NetworkMiner was the list of genes identified in the RNASeq experiment ranked by the stat parameter of the DeSeq2 analysis. We used the "All ppis" interactome (genes version) and allowed one external intermediate protein.

The FatiGO algorithm [19] was applied to perform the functional enrichment analysis of the proteins in each of the networks (excluding orphan nodes). We run FatiGO using GO terms (biological processes) as annotation and the interactome used for the NetworkMiner analysis as the reference list of genes. A value of $p$-value adjusted by $\operatorname{FDR}<0.05$ was considered for statistical significance.

\subsection{Flow cytometry}

Cells were harvested by trypsinization, fixed overnight in 70\% ethanol, washed and incubated for $1 \mathrm{~h}$ in PBS containing $100 \mu \mathrm{g} / \mathrm{mL}$ RNAse A, $10 \mu \mathrm{g} / \mathrm{mL}$ propidium iodide (PI) and 0.05 Nonidet P-40\%. The total cell number and the percentage of cells in G0-G1, S or G2-M phases was counted using standard flow cytometry methods and a BD FACSCanto II flow cytometer (BD Biosciences).

\subsection{Wound closure assay}

Wild type or Tnfrsf $12 a^{-1-}$ VSMCs were grown to confluence in growth medium and then serum deprived for $24 \mathrm{~h}$. A single scrape wound was made, and cells were then incubated with or without rTWEAK (100 ng/mL). 10\% FBS was used as a positive control. Migration into the denuded area was monitored by photomicroscopy. To inhibit VSMCs proliferation, cells were preincubated $30 \mathrm{~min}$ with an antiproliferative dose of Actinomycin D $(0.01 \mu \mathrm{g} / \mathrm{mL}$; Sigma) [20] before rTWEAK stimulation. 
a

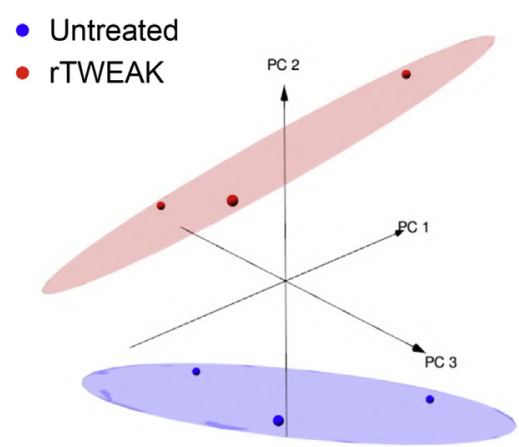

C

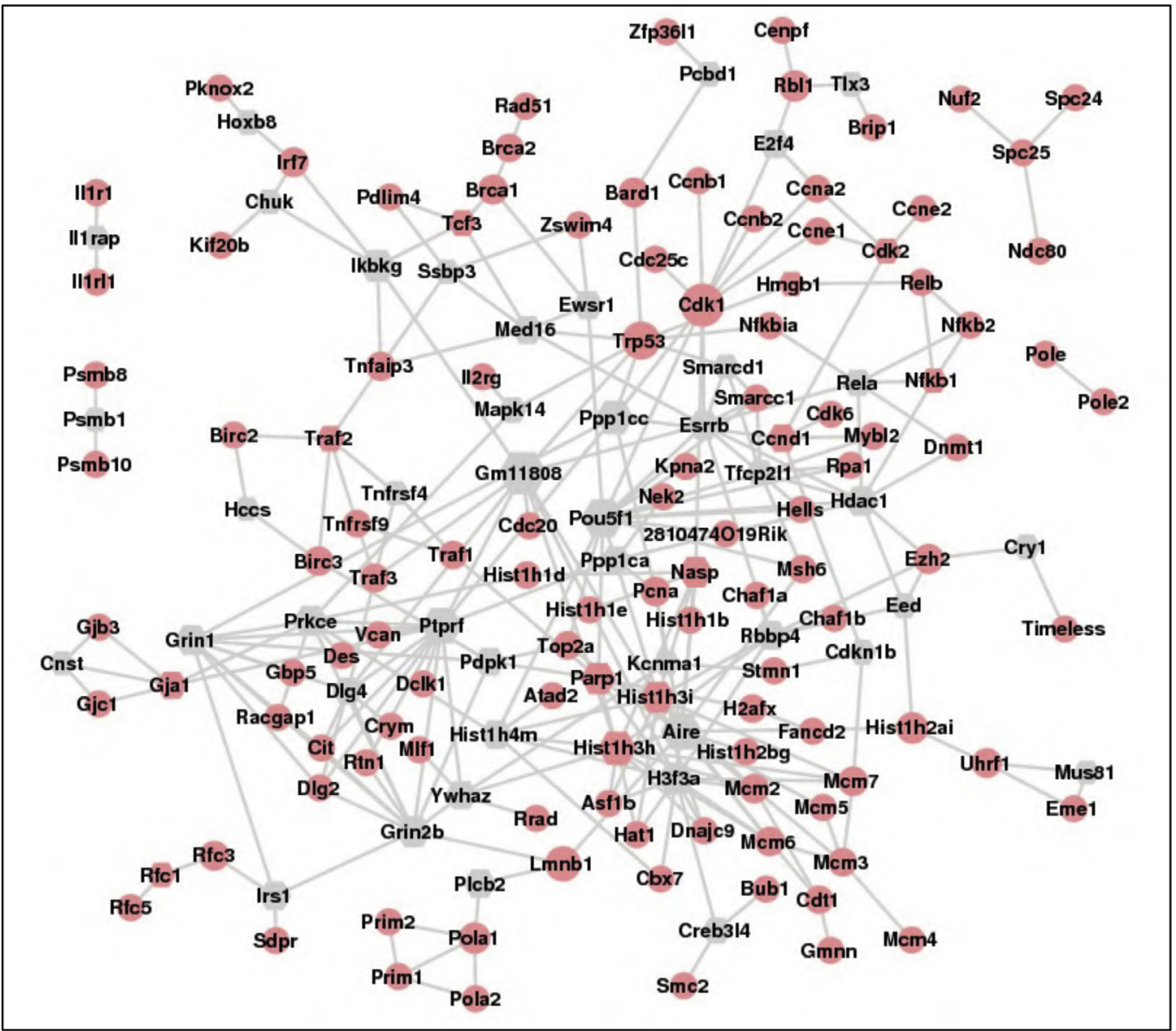

d

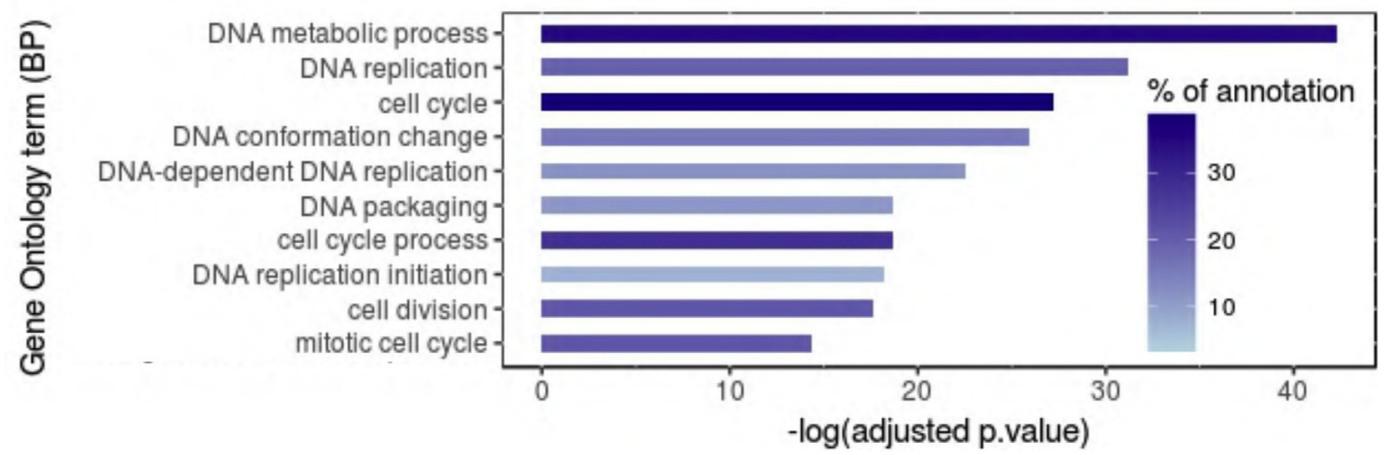

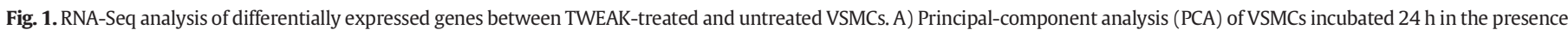

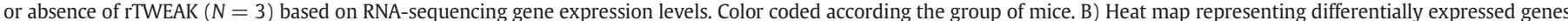

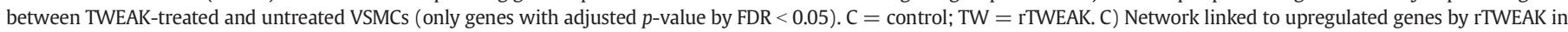

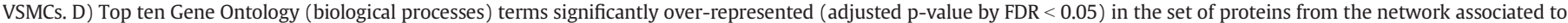
TWEAK upregulated genes. 
a

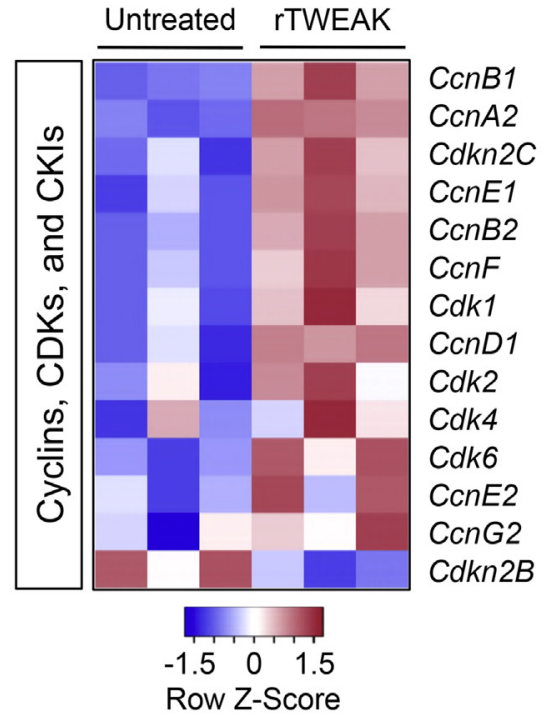

b

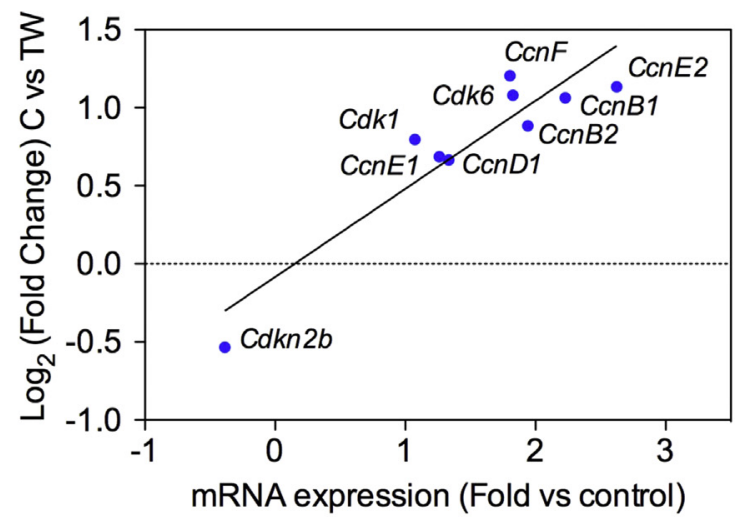

C

Control

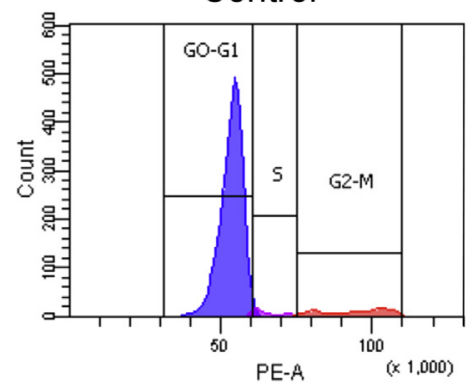

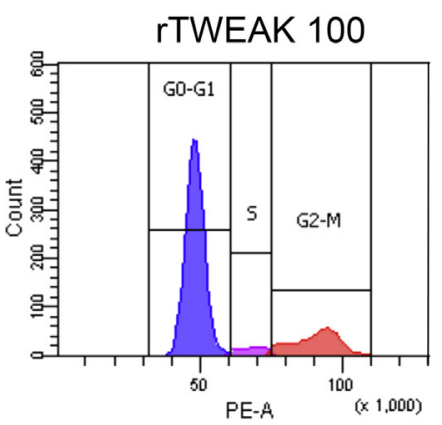

$10 \%$ FBS

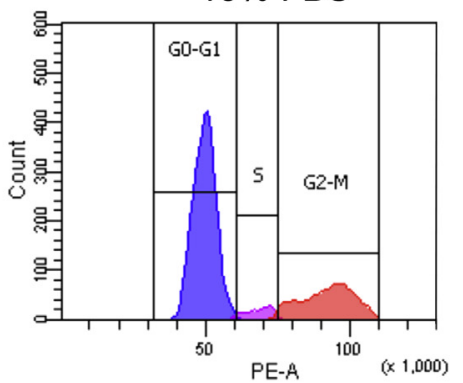

d

e
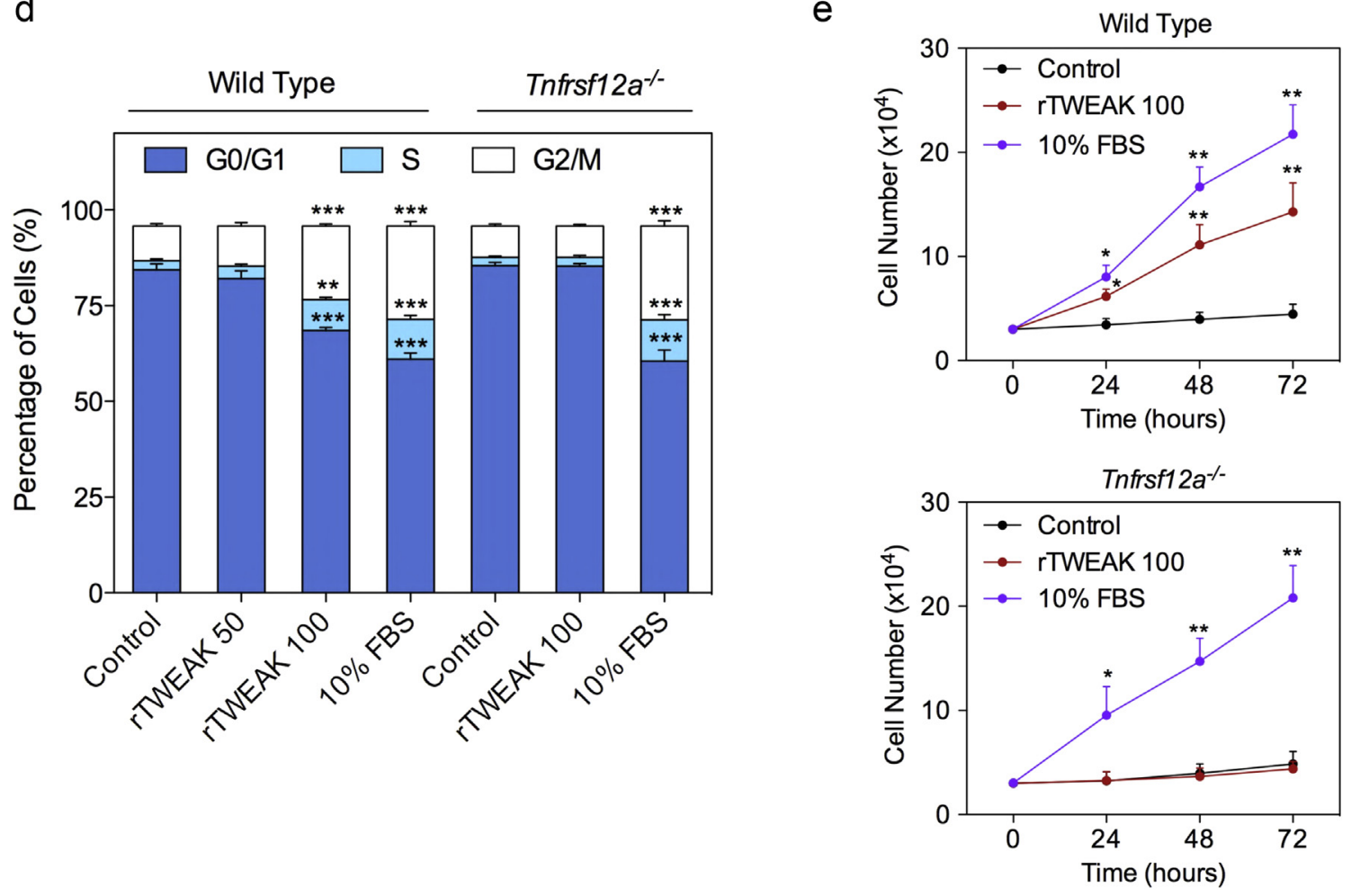

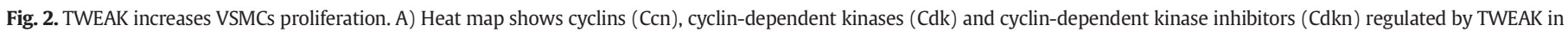

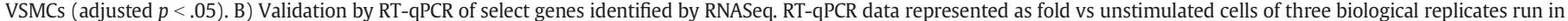

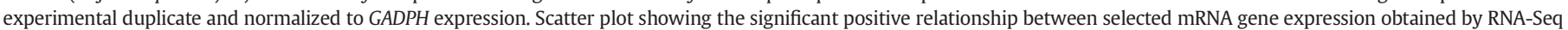

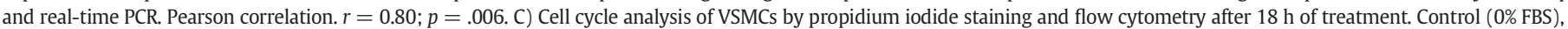

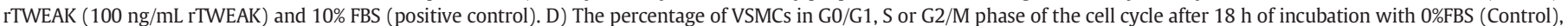

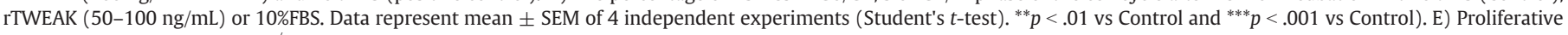

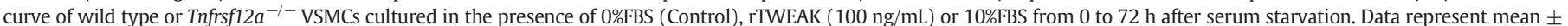
SEM of 3 independent experiments (Student's $t$-test) ${ }^{*} p<.05$ vs Control and ${ }^{* *} p<.01$ vs Control). 
a
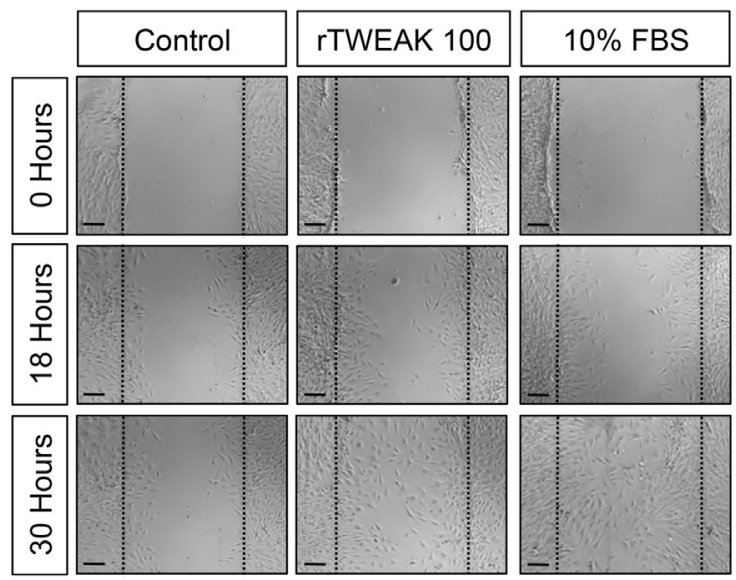

b

Wild Type
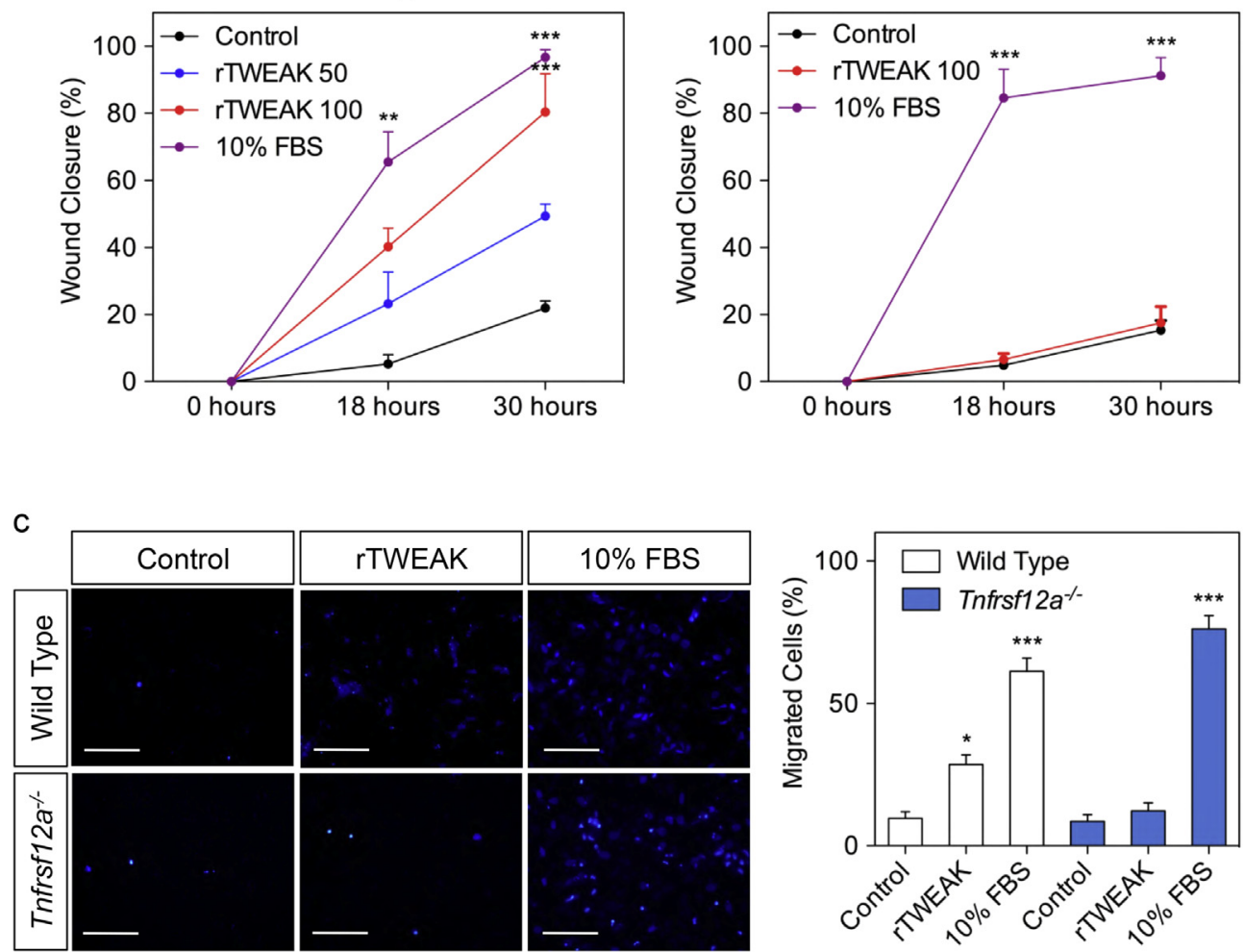

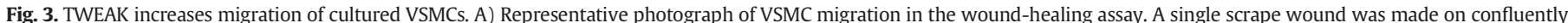

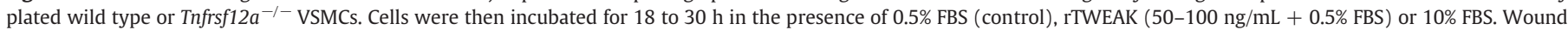

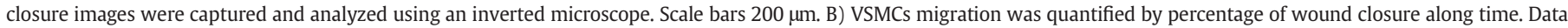

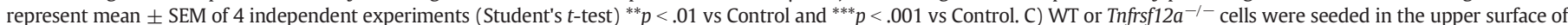

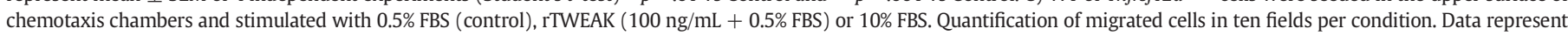
the mean \pm SEM of 4 independent experiments (Student's $t$-test) ${ }^{*} p<.05$ vs Control and ${ }^{* * *} p<.001$ vs Control. Scale bars $20 \mu$ m.

\subsection{Migration assay}

Migration of Wild type or Tnfrsf $12 a^{-/-}$VSMCs was measured in $8 \mu \mathrm{m}$ pore transwell 24-well cell culture inserts (Costar). Cells containing $0.2 \% \mathrm{BSA}$ in DMEM were seeded $\left(70 \times 10^{4}\right.$ per well) into the migration chamber. The lower wells of the chemotaxis chambers were filled with rTWEAK ( 50 or $100 \mathrm{ng} / \mathrm{mL}$ ) plus $0.5 \%$ of FBS. $10 \%$ FBS was used as positive control of migration. After $4 \mathrm{~h}$ of nonmigrating cells were removed from the upper part of the chamber and, the nuclei of migrated cells was fixated and stained with DAPI. The number of migrated cells was counted in 10-randomly selected fields by Nikon Eclipse E400 fluorescence microscope.

\subsection{RNA extraction and real-time $P C R$}

Femoral tissues were snap frozen in N2 liquid and homogenates were resuspended for mRNA analysis. Total RNA from VSMC or femoral arteries was obtained by TRIzol method (Life Technologies) and quantified by absorbance at $260 \mathrm{~nm}$ in duplicate. $2 \mu \mathrm{g}$ of total RNA was reverse transcribed according to the manufacturer's protocol (Applied Biosystems). Real-time PCR was performed on a TaqMan ABI 7700 Sequence Detection System using specific TaqMan probe for 18S (VICTAMRA 431089E), Tnfrsf12a (Mm_00489103_m1) and Tnfsf12 (Mm 02583406_s1) or SYBR Green (Takara Biotechnology) depend of the studied gene. PCR primers for SYBR Green are available in the data 
a
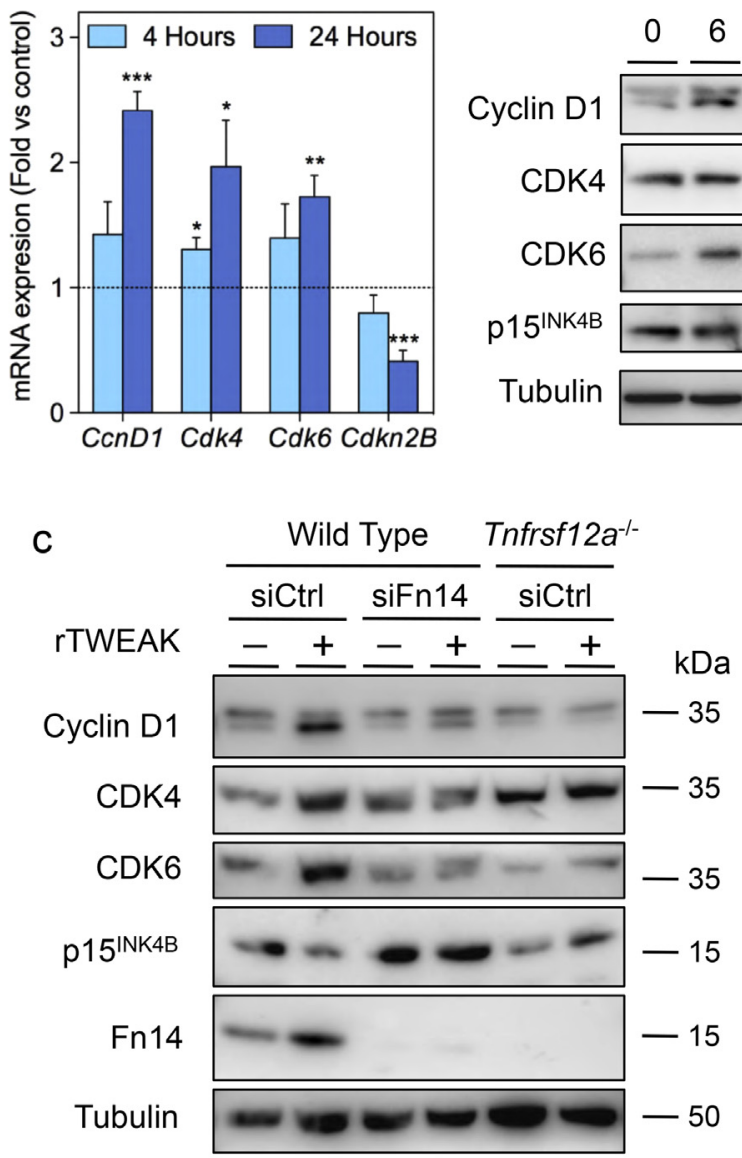

d

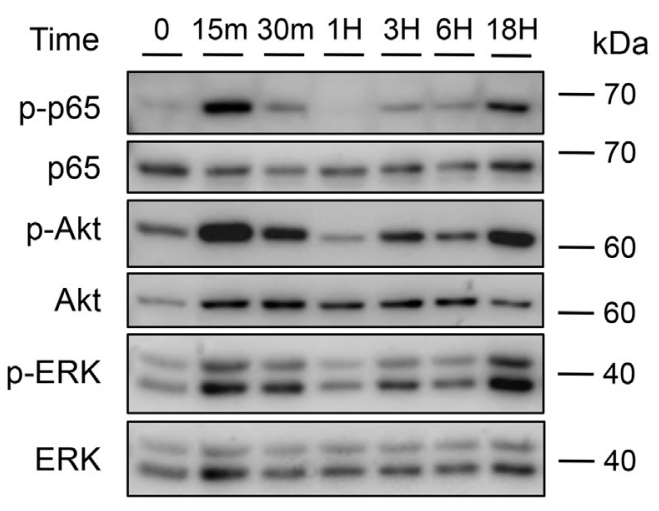

e

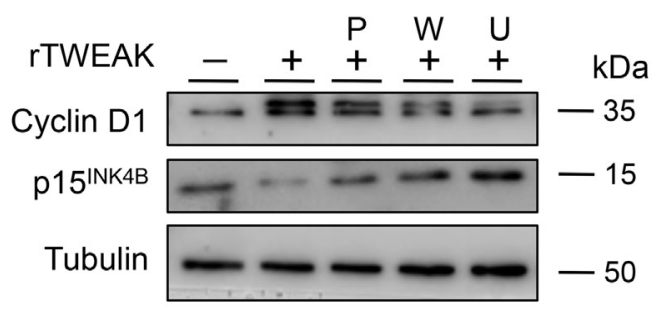

b
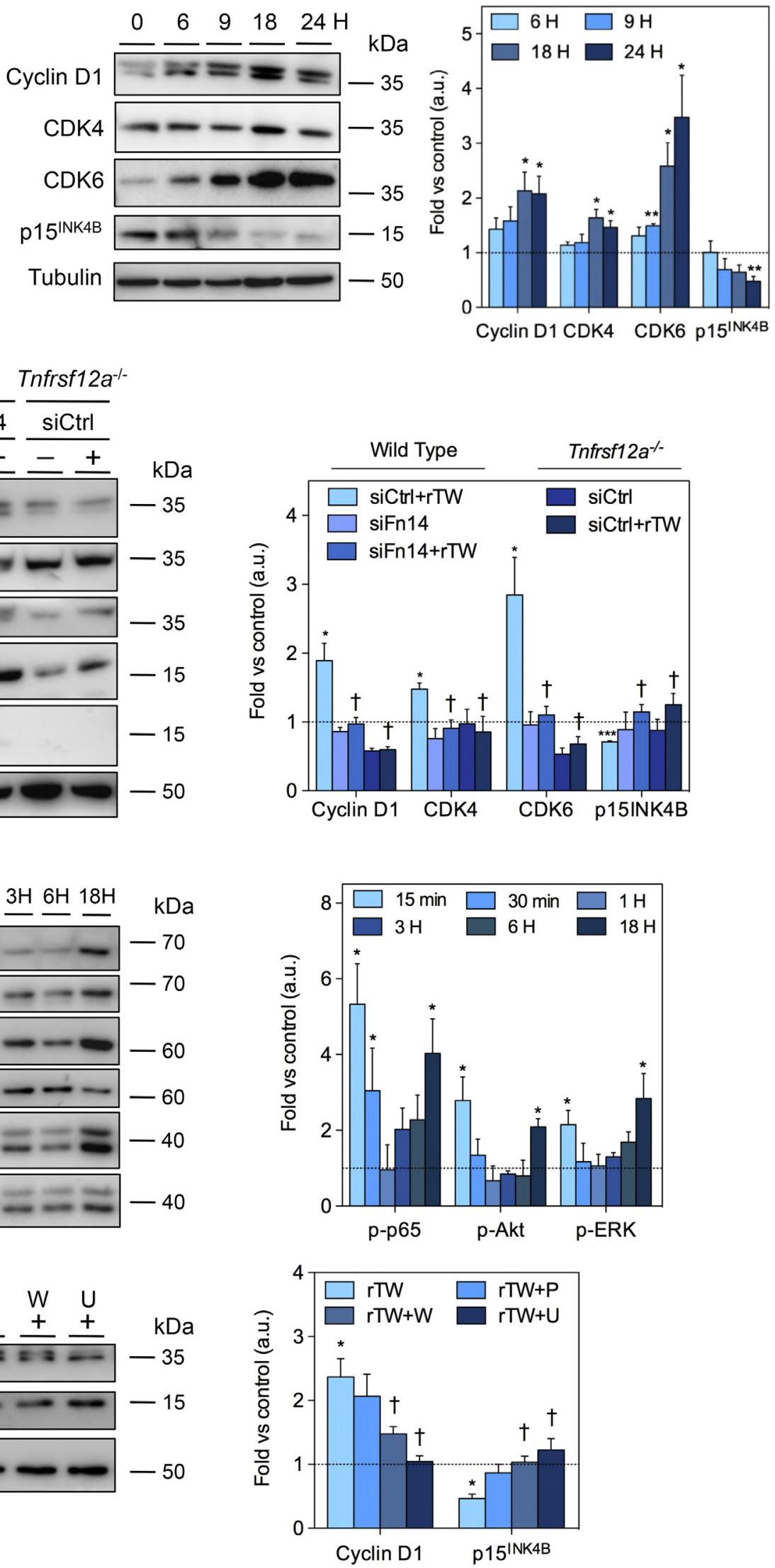
supplemental (Supp. Table 1). 18S rRNA served as housekeeping gene for TaqMan studies and Gapdh for SYBR. Housekeeping genes were amplified in parallel with the genes of interest. All measurements were performed in triplicate. The amount of target mRNA in samples was estimated by the $2 \Delta C T$ relative quantification method. Values of each sample were obtained as multiples of their baseline values.

\subsection{Western-blot}

Cultured murine VSMCs from WT or Tnfrsf $12 a^{-/-}$mice in the different experimental conditions were collected in ice-cold buffer containing 50 mM Tris- $\mathrm{HCl}$ pH 7.4, $150 \mathrm{mM} \mathrm{NaCl}, 2$ mM EDTA, 2 mM EGTA, 0.2\% Triton X-100, 0.3\% NP-40, $0.2 \mathrm{mM}$ PMSF, $0.2 \mathrm{mM} \mathrm{Na} 3 \mathrm{VO} 4$ and 10 $\mu \mathrm{L} / \mathrm{mL}$ of phosphatase inhibitor cocktail (P0044 Sigma) and pelleted. After normalizing for equal protein concentration, cell lysates were resuspended in SDS sample buffer before separation by SDS-PAGE. Following transfer of the proteins onto nitrocellulose membranes and probed using the following antibodies: anti-Fn14 antibody (1:1000; EPR3179, Abcam), anti-p15 ${ }^{\text {INK4B }}$ (1:500; AV03047, Sigma), anti-Cdk4 (1:1000; ab137675, Abcam), anti-Cdk6 (1:1000; sc53638, Santa Cruz Biotechnology), anti-Cyclin D1 (1:1000; s18396, Santa Cruz Biotechnology) and anti-alpha-tubulin (1:10000; T5168, Sigma). After incubation with appropriate HRP-conjugated secondary antibody (Jackson Laboratory), proteins were visualized by ECL Western Blotting Detection Reagents (Amersham Biosciences) according to manufacturer instructions. Densitometry analysis of the gels was carried out using ImageJ software from the NIH (http://rsbweb.nih.gov/ij/).

\subsection{Human samples}

Stented [21] and non-stented coronary arteries [22] were isolated from hearts of cardiac transplant recipients. Coronary arteries were classified according to their localization, atheromatous status, nature of the donor, and then stored in a biobank (INSERM U1148, Bichat hospital, Paris, France) [23]. The Institutional Review Board, IRB 0006477 of Hôpitaux Universitaires Paris-Nord Val de Seine, Paris7 University, and Assistance Publique-Hôpitaux de Paris approved the use of explanted hearts for research. Human healthy coronary arteries were collected from freshly excised hearts removed during transplant operations at the Hospital de la Santa Creu I Sant Pau (Barcelona, Spain). The local ethics committee approved the use of healthy coronary arteries.

\subsection{Wire injury model}

Animal procedures were strictly in accordance with the Directive 2010/63/EU of the European Parliament and were approved by the Institutional Animal Care and Use Committee of IIS-Fundación Jiménez Díaz. Tnfrsf12a-knockout mice, Tnfsf12 knockout mice, and wild-type (WT) counterparts (generously provided by Biogen, Inc.) have been reported previously and backcrossed onto the C57BL/6 strain [24,25].

Male mice aged 10 weeks were anesthetized by ketamine/xylazine and endoluminal injury to the common femoral artery was performed by three passages of a $0.25-\mathrm{mm}$ diameter angioplasty guidewire (ev3 ${ }^{\mathrm{TM}}$, Medtronic) as previously described [26]. To study the effect of anti-TWEAK treatment, 10 weeks-old wild type mice were divided in two groups: Mice injected i.p. with anti-TWEAK $\mathrm{mAb}(10 \mathrm{mg} / \mathrm{kg}$ twice a week) or an irrelevant isotype matched control $\operatorname{IgG}$ specific for Hen egg lysozyme (10 mg/kg twice a week). Biogen, Inc. generously provided both anti-TWEAK mAb (clone P2D10; mIgG2a generated by immunizing TWEAK-deficient mice on C57BL/6 background with recombinant human TWEAK) and irrelevant isotype matched control IgG specific for Hen egg lysozyme.

\subsection{Morphometric analysis}

The femoral artery was excised from the inguinal ligament to the branching of the profundal femoris artery and stored in paraformaldehyde for $24 \mathrm{~h}$ and later in ethanol until paraffin embedded. Femoral arteries were cut into 5 - $\mu \mathrm{m}$ serial sections, and 6 cross sections taken at regular intervals throughout the artery were stained with hematoxylin and eosin. For the morphometric analyses, Leica DMD 108 microscopy was used to measure the external elastic lamina, internal elastic lamina, and lumen circumference to calculate the medial- and neointimal area and the neointima/media ratio 14 days after dilation.

\subsection{Immunohistochemistry}

Immunohistochemical analysis was carried out as previously described [13]. For mice tissues, primary antibodies were the anti-smooth muscle cell markers alpha smooth muscle actin (clone 1A4, F3777, Sigma) and calponin (1:100; ab46794 Abcam), anti-TWEAK (1:50; NBP1-6774 Novus), anti-Fn14 (1:50; 4403S Cell Signalling), anti-cyclin D1 (1:50; 92G2 Cell Signalling), anti-CDK4 (1:100; ab137675 Abcam), anti-CDK6 (1:100; GTX103992 GeneTex), anti-p15 ${ }^{\text {INK4B }}(1: 100$; AV03047 Sigma), the proliferation marker anti-PCNA (1:200; sc-7907, Santa Cruz Biotechnology), T-lymphocyte marker anti-CD3 (1:500; A0452 Dako), macrophage marker anti-CD68 (1:200; ab53444 Abcam), endothelial cell marker anti-CD31 (1:50; ab28364 Abcam) and fibroblast marker anti-S100A4 (1:50; ab93283 Abcam). Donkey anti-goat biotin, donkey anti-rabbit biotin and, goat anti-rat biotin (Amersham) was used as secondary antibodies, ABComplex/HRP was then added and sections. Immunohistochemistry color was developed with DAB (Dako), and section were counterstained with hematoxylin, and mounted in DPX (Millipore). Incubation without primary antibodies and/or irrelevant species and isotype-matched immunoglobulins was performed as a negative control for all immunostaining studies. Computer-assisted morphometric analysis was performed with the Image-Pro Plus software (version 4.5.0 for Windows) in a blinder manner. The threshold setting for area measurement was equal for all images. Results were expressed as \% positive area of, Cyclin D1, CDK4, CDK6 and, p15 $5^{\mathrm{INK} 4 \mathrm{~B}}$ in total artery, and as \% of PCNA positive cells versus total cells.

For human tissues, primary antibodies were anti- $\alpha$-SMA (1:500; ab5694 Abcam), anti-calponin (1:100; ab46794 Abcam), anti-TWEAK (1:100; ab37170 Abcam), anti-Fn14 (1:50; ab109365 Abcam) and anti-PCNA (1:200; sc-7907, Santa Cruz Biotechnology).

\subsection{Statistical analysis}

Animal sample size for each study was chosen based on literature documentation of similar well-characterized experiments. The number of animals in each study is listed in the figure legends. Values are expressed as mean \pm SEM ( $\mathrm{n}$ is noted in the figure legends). In vitro experiments were replicated at least 3 times unless otherwise noted. Statistical differences were measured using two-sided Student's test or a one-way ANOVA followed by a post hoc Bonferroni pairwise comparison test. A nonparametric test (Mann-Whitney) was used when data

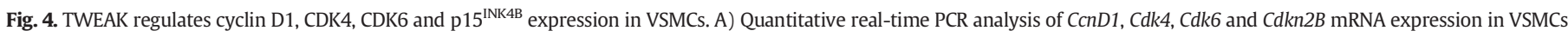

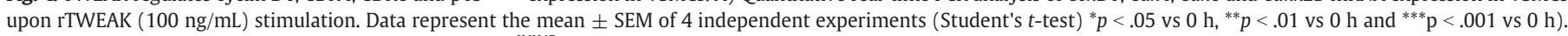

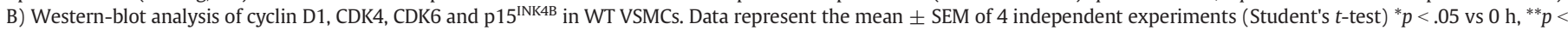

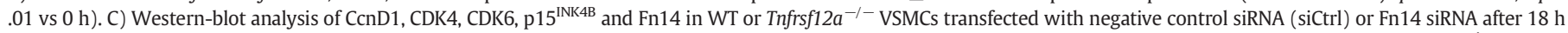

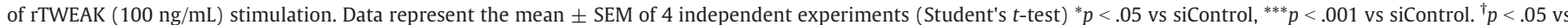

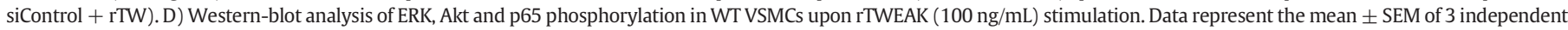


represent the mean $\pm \mathrm{SEM}$ of 3 independent experiments (Student's $t$-test) ${ }^{*} p<.05$ vs control; ${ }^{\dagger} p<.05$ vs rTW. 
a

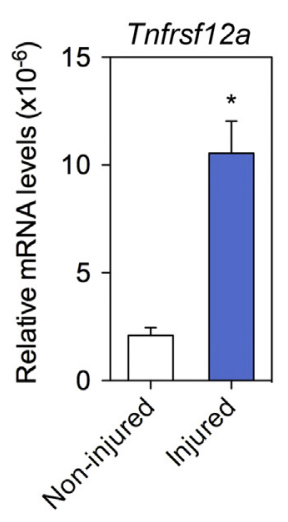

b

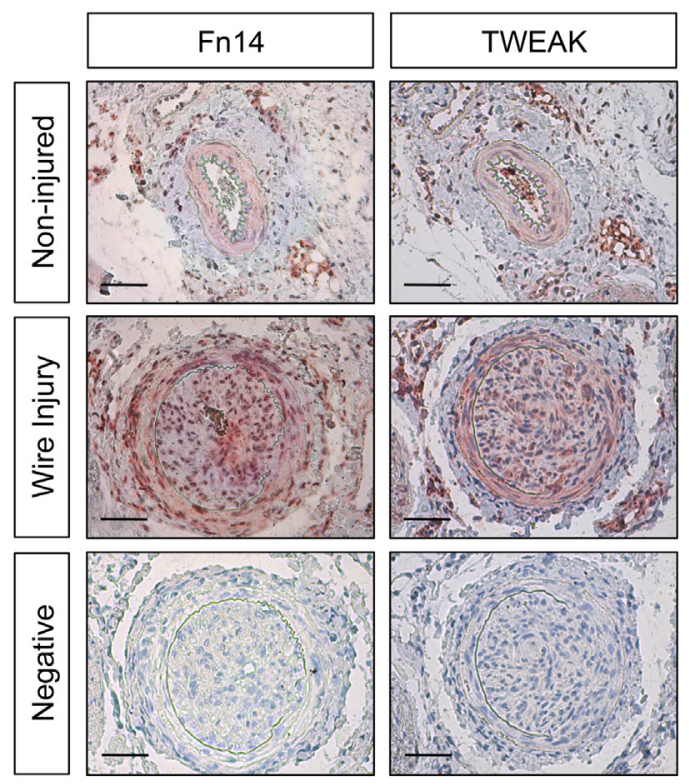

C
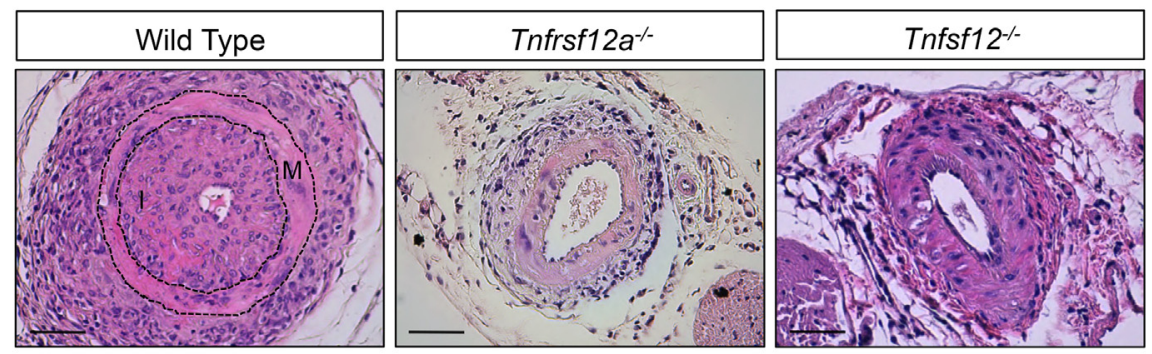

d
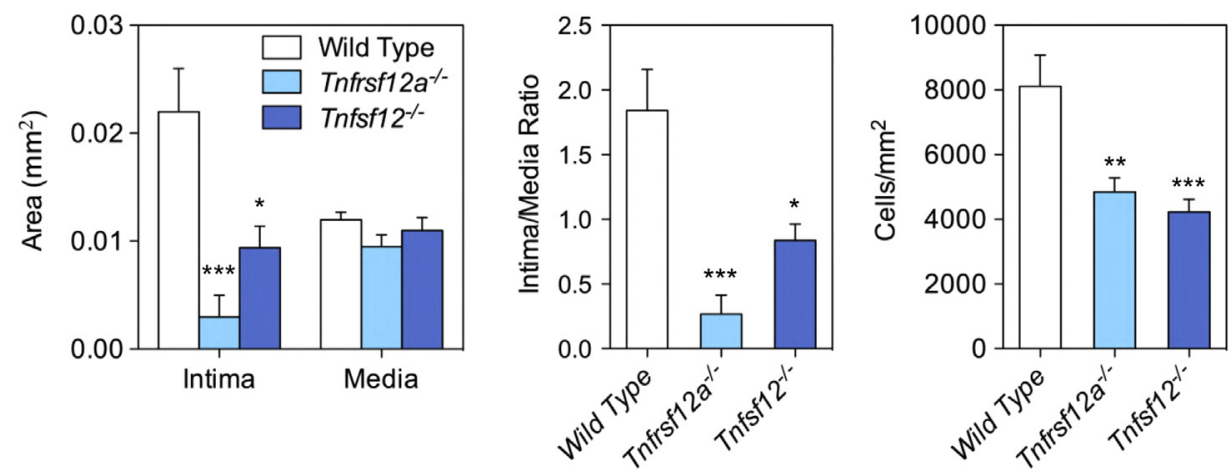

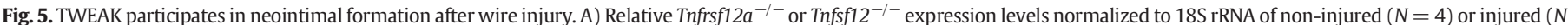

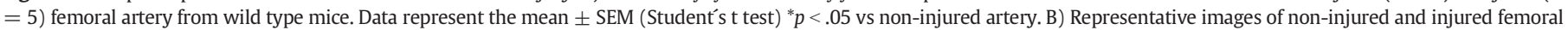

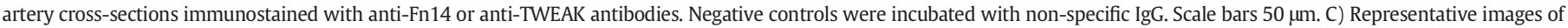

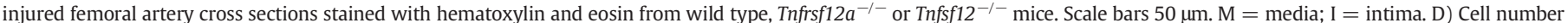

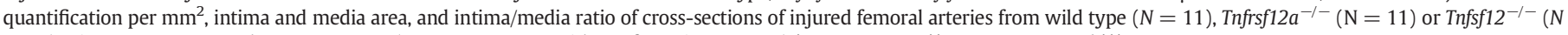
$=10$ ) mice. Data represent the mean \pm SEM (One-way ANOVA with Bonferroni's post-test) ${ }^{*} p<.05$ vs WT, ${ }^{* *} p<.01$ vs WT and ${ }^{* * *} p<.001$ vs WT.

did not pass the normality test. A value of $p<.05$ was considered statistically significant. Data analysis was performed using GraphPad Prism Software Version 7 (GraphPad, San Diego, CA, USA).

\subsection{Data statement}

Datasets have been deposited in NCBI Gene Expression Omnibus and are accessible through GEO series number GSE114116 (https://www. ncbi.nlm.nih.gov/geo/query/acc.cgi?acc=GSE114166).

\section{Results}

3.1. TWEAK modulates the expression of genes associated with cell proliferation in VSMCS

We initially aimed to identify genes that are modulated by TWEAK in VSMCs. To this end, murine aortic VSMCs were incubated in the presence or absence of murine recombinant TWEAK (rTWEAK; $100 \mathrm{ng} / \mathrm{mL}$ ) for $24 \mathrm{~h}$. The dose of rTWEAK was selected based in our 
a
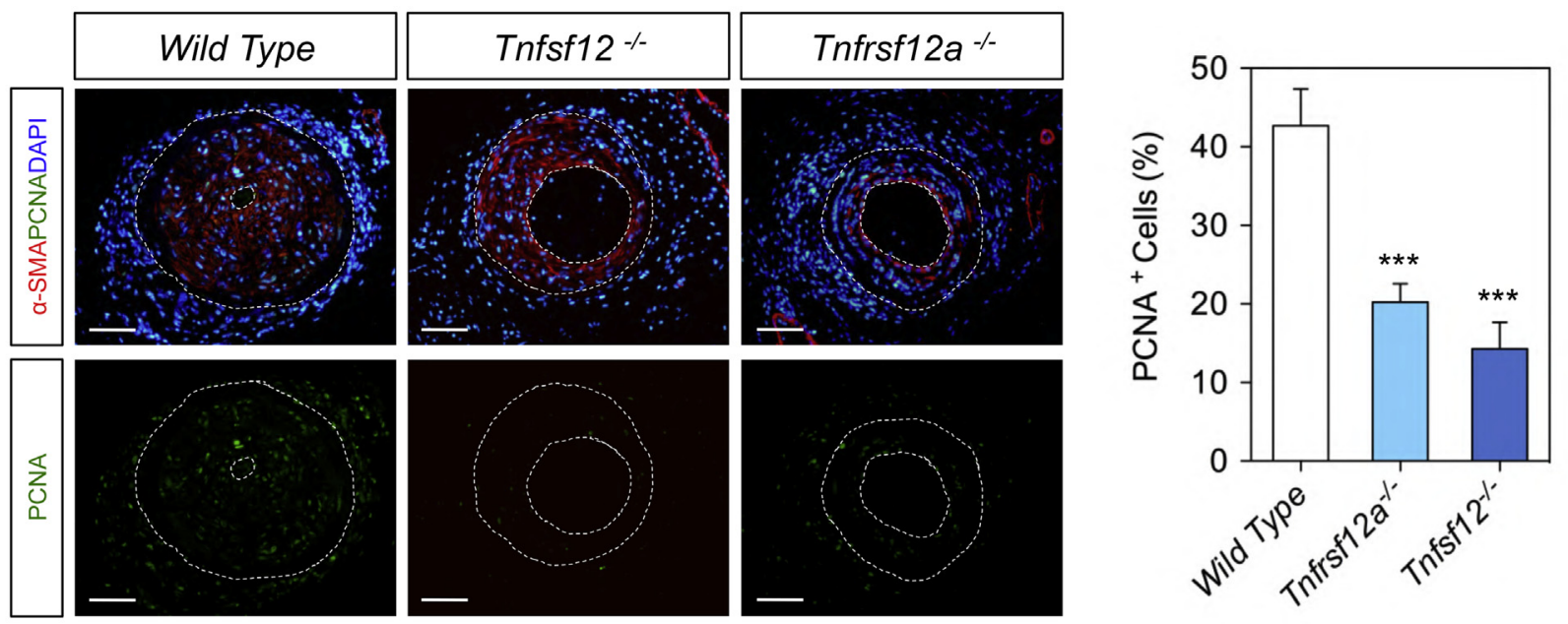

b
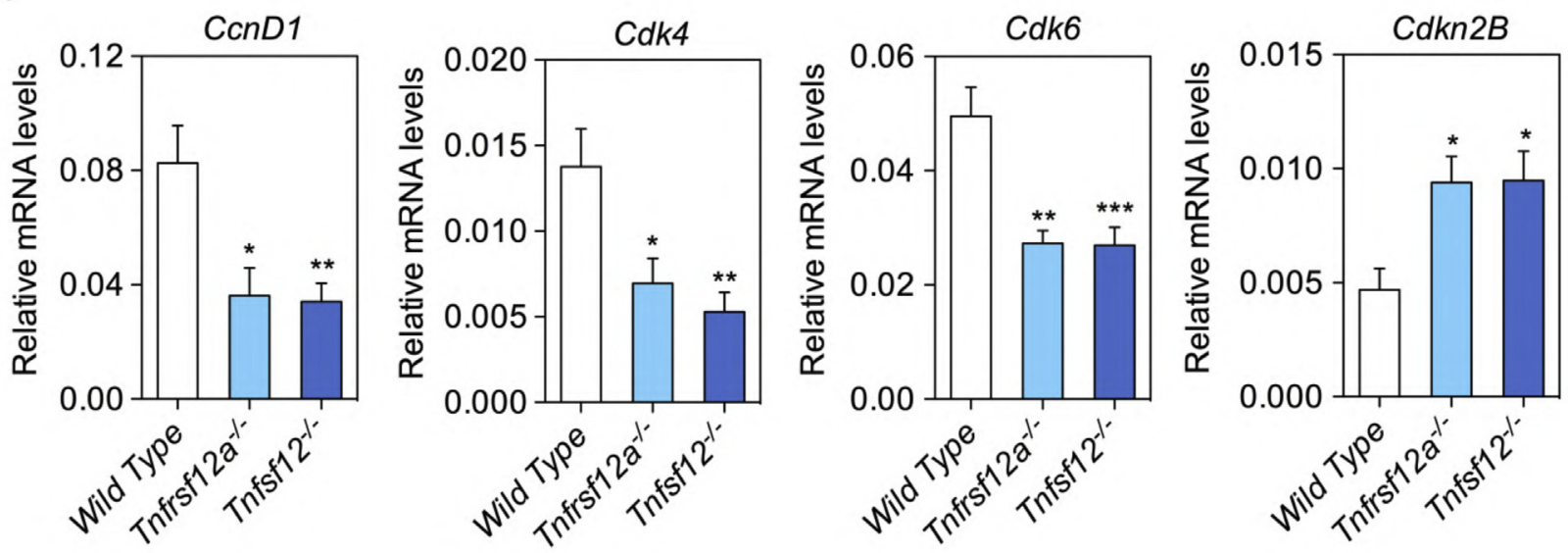

C
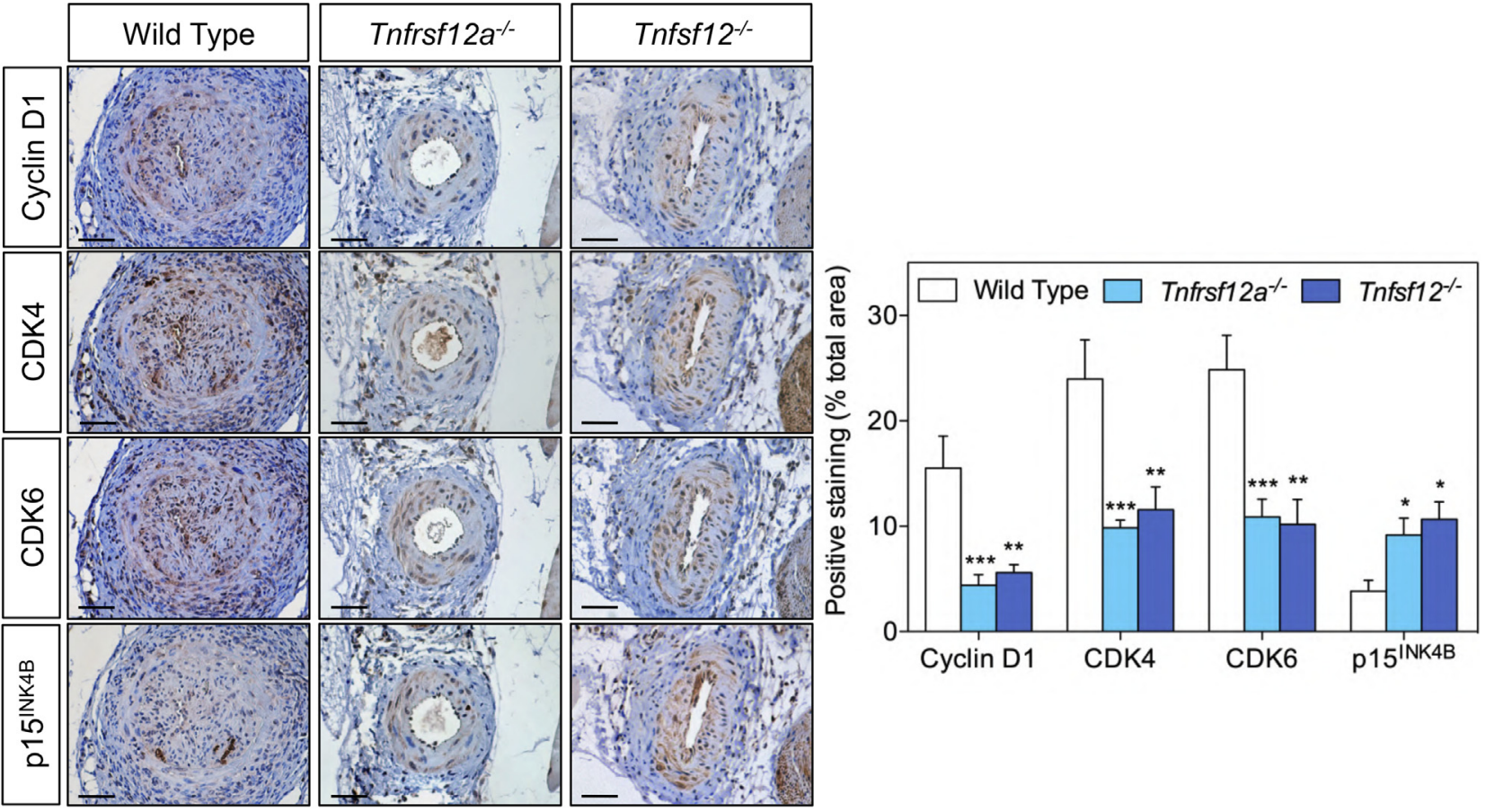
previous studies $[10,13]$. The RNA Sequencing (RNA-Seq) transcriptome profiling analysis revealed 2702 genes differentially expressed in VSMCs under rTWEAK stimulation, 1611 up-and 1091 downregulated (Fig. 1). The number of significant up and down-regulated genes exceeds the range where a classical functional enrichment method can characterize gene signatures. Instead, we performed a gene set enrichment analysis that reported 984/1032 biological processes, 10/10 molecular functions, 6/6 cellular components and 62/92 KEGG pathways over-represented in rTWEAK stimulated versus control conditions (Suppl. File 1). We performed a gene set-like network enrichment analysis using NetworMiner algorithm in order to capture the most relevant functional modules, that are up/down regulated by rTWEAK in VSMCs [18]. This method extracts functional modules associated to a phenotype by evaluating both, the topological robustness of a protein-protein interaction network and the link of its components to a condition. The modules are presented as protein networks and the level of significance is obtained from a comparison to networks generated from random lists of genes. Remarkably, the resultant network may contain proteins not necessarily labeled as associated to the phenotype by the expression analysis but important in the internal structure of the functional module.

We obtained two significant protein networks linked to genes up and down-regulated by TWEAK. The network associated to the upregulation by rTWEAK in VSMCs (Fig. 1C) contains 159 connected proteins (116 significantly up-regulated in the expression analysis) that are enriched in Gene Ontology terms related to cell proliferation (Fig. 1D and Suppl. Table 2). We also found a smaller but significant network of down-regulated genes associated with several biological overrepresented processes related to DNA binding and histone regulation, development processes and cell cycle (Fig. S2 and Suppl. Table 3).

$\mathrm{G}_{1}$ to $\mathrm{S}$ cell cycle progression is modulated by the balance between positive cell cycle regulators [cyclins and cyclin-dependent kinases (CDK)] on one hand and CDK inhibitors (CDKI) on the other [27]. Fig. 2A shows a heatmap of differentially expressed cyclins, CDK and CDKI in VSMCs incubated in the presence or absence of rTWEAK. To confirm the results from the RNA-Seq, a subset of different genes associated with cell proliferation ( $C \mathrm{C} n \mathrm{~B} 1, B 2, D 1, E 1, E 2$, and $F, C d k 1, C d k 4$ and $C d k 6$, and $C d k n 2 b$ ) were independently validated using RT-qPCR (Suppl. Table 4). We observed an excellent correlation between RNA-Seq and RT-PCR findings ( $r=0.80 ; p=.006$; Pearson correlation; Fig. 2B). Interestingly, $C d k n 2 b$, a highly conserved cell-cycle regulator and tumor suppressor gene, was consistently downregulated by TWEAK in VSMCs (Fig. 2A-B). Collectively, these data suggest that TWEAK signaling contributes to VSMCs proliferation. Therefore, we decided to analyze the role of TWEAK on cell proliferation both in vitro and in vivo.

\subsection{TWEAK increases VSMCs proliferation and migration}

We analyzed Fn14 expression in VSMCs and we observed that $\alpha$ SMA positive VSMCs express Fn14 (Supp. Fig. S1B-C). To determine whether TWEAK regulates VSMCs proliferation, we performed cell cycle analysis of rTWEAK-stimulated VSMCs by measuring the DNA content of cells stained with propidium iodide. Cells growing in 10\% FBS were used as a positive control of cell proliferation. There was a decrease of cells in G0/G1 phase and an increase of cells in the S and G2/M phase in rTWEAK-stimulated VSMCs at $18 \mathrm{~h}$ compared with control cells (Fig. 2C-D). However, rTWEAK was unable to induce the transition of cells from G0/G1 to S phase in VSMCs from Tnfrsf12a deficient mice (Tnfrsf $12 a^{-1-}$ ) (Fig. 2C-D), indicating that TWEAK activates cell proliferation through its functional receptor Fn14. Consistently, cell number increased over the time when VSMCs were incubated in the presence of rTWEAK (Fig. 2E). No effect was observed in VSMCs lacking the Fn14 receptor (Fig. 2E).

We next performed scratch wound healing assays in VSMCs from wild type (WT) and Tnfrsf $12 a^{-1-}$ mice to analyze whether TWEAK plays a role in cell migration. rTWEAK-stimulated VSMCs from WT mice efficiently reoccupied the scratched area, whereas Tnfrsf $12 a^{-1-}$ VSMCs did not migrate in response to rTWEAK (Fig. 3A-B). To confirm that TWEAK induces VSMCs migration, cells were preincubated $30 \mathrm{~min}$ in the presence or absence of anti-proliferative and nonapoptotic concentration of actinomycin D (ActD) [20]. rTWEAKstimulated VSMCs also reoccupied the scratched area in presence of ActD indicating that TWEAK induces VSMCs migration (Fig. S3). Moreover, WT VSMCs efficiently migrated in transwell assays whereas similarly treated Tnfrsf $12 a^{-1-}$ VSMCs did not (Fig. 3C). Altogether, our results demonstrate that TWEAK-induced VSMCs proliferation and migration is dependent on its binding to Fn14.

We subsequently screened the expression of TWEAK-regulated genes identified by RNA-seq to gain more mechanistic insight into the proliferative effects of TWEAK. As commented above, Cdkn2b ( $\left.15^{\mathrm{INK} 4 \mathrm{~B}}\right)$, a highly conserved cell-cycle regulator and tumor suppressor gene, was consistently downregulated by TWEAK in the RNA-Seq and qRT-PCR studies. Time-kinetic studies assessed in WT VSMCs showed that rTWEAK downregulated $C d k n 2 B$ mRNA expression and $\mathrm{p} 15^{\mathrm{INK} 4 \mathrm{~B}}$ protein level in a time-dependent manner (Fig. 4A-B). In addition, the downregulation of $\mathrm{p} 15^{\mathrm{INK} 4 \mathrm{~B}}$ was dependent of the presence of Fn14, since TWEAK failed to downregulate $\mathrm{p} 15^{\mathrm{INK} 4 \mathrm{~B}}$ protein level in siRNA Fn14-transfected VSMCs or Tnfrsf $12 a^{-1-}$ VSMCs (Fig. 4C).

The transition of $G_{1}$ to $S$ phase is regulated by $D$-type cyclins and it has been demonstrated that $\mathrm{p} 15^{\mathrm{INK} 4 \mathrm{~B}}$ is an inhibitor of CDK4/CDK6 and cyclin D1 expression in VSMCs [28]. Therefore, we have analyzed CcnD1 (cyclin D1), Cdk4 and Cdk6 mRNA expression in rTWEAK-stimulated VSMCs. In line with Mathew et al. [28], rTWEAK increased CcnD1, $C d k 4$ and $C d k 6$ mRNA expression (Fig. 4A). In addition, rTWEAK increased cyclin D1, CDK4 and CDK6 proteins levels in a time-dependent manner (Fig. 4B). The upregulation of cyclin D1, CDK4 and CDK6 was also dependent of Fn14 since TWEAK failed to upregulate these proteins in Tnfrsf $12 a^{-1-}$ VSMCs or wild type cells transfected with siRNA against Fn14 (Fig. 4C).

\section{3. $p 15^{I N K 4 B}$ and cyclin D1 expression is regulated by ERK1/2 and Akt in VSMCS}

To analyze the mechanism/s by which TWEAK regulates $\mathrm{p} 15^{\mathrm{INK} 4 \mathrm{~B}}$ and cyclin D1 expression in VSMCs, we studied signal pathways that control cell proliferation. TWEAK induced phosphorylation of ERK1/2, Akt and p65, a subunit of nuclear factor kappa B (NF-kB), in a timedependent manner, peaking at $15 \mathrm{~min}$ and $18 \mathrm{~h}$ (Fig. 4D). To analyze the role of ERK1/2, Akt and NF-kB on p15 ${ }^{\mathrm{INK} 4 \mathrm{~B}}$ and cyclin D1 expression, we used the specific inhibitor U0126 (ERK1/2), wortmannin (PI3K) and partthenolide (NF-kB). The inhibitors of ERK1/2 (UO126) and Akt (wortmannin) prevented $\mathrm{p} 15^{\mathrm{INK} 4 \mathrm{~B}}$ downregulation and cyclin D1

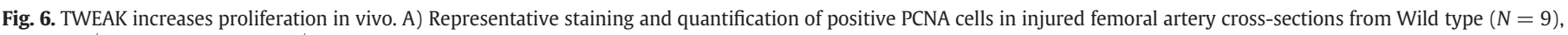

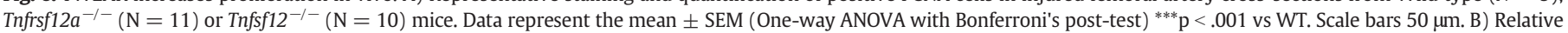

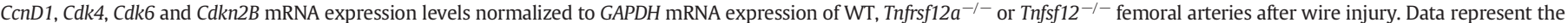

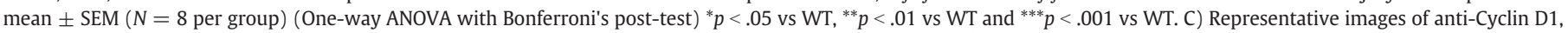

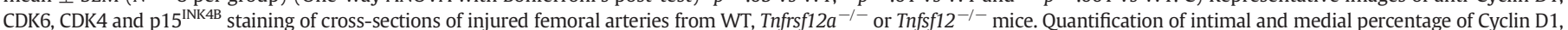

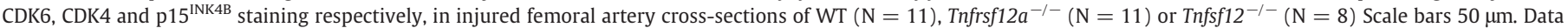
represent the mean \pm SEM (One-way ANOVA with Bonferroni's post-test) ${ }^{*} p<.05$ vs WT, ${ }^{* *} p<.01$ vs WT and ${ }^{* * *} p<.001$ vs WT. 
a
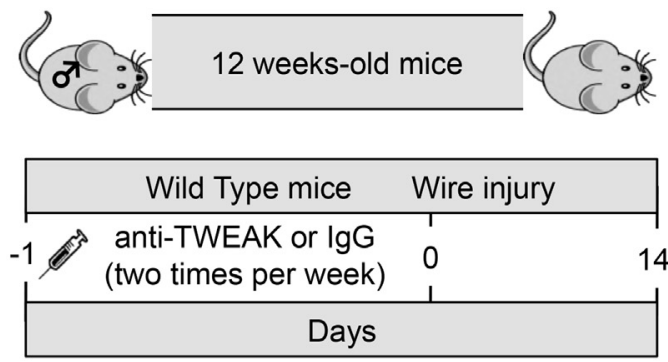

b
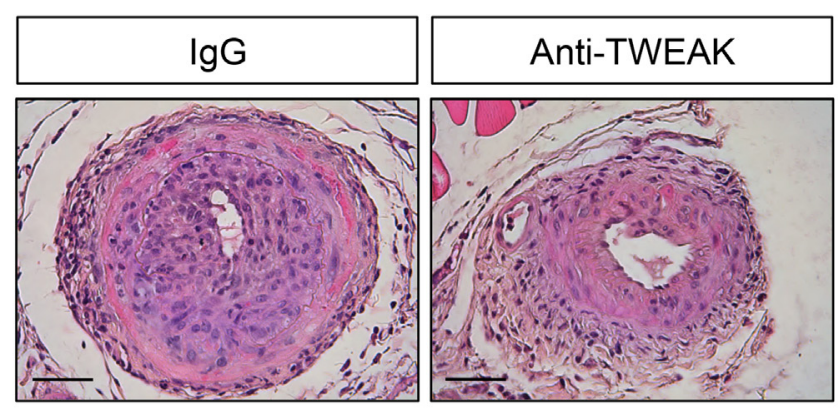

C
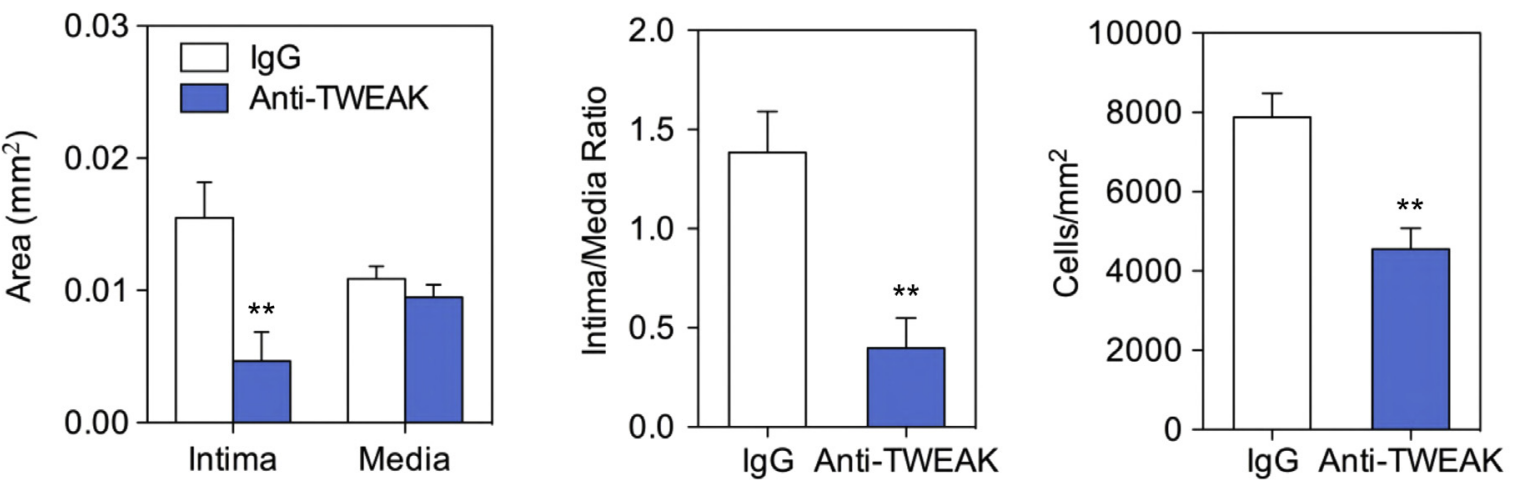

d
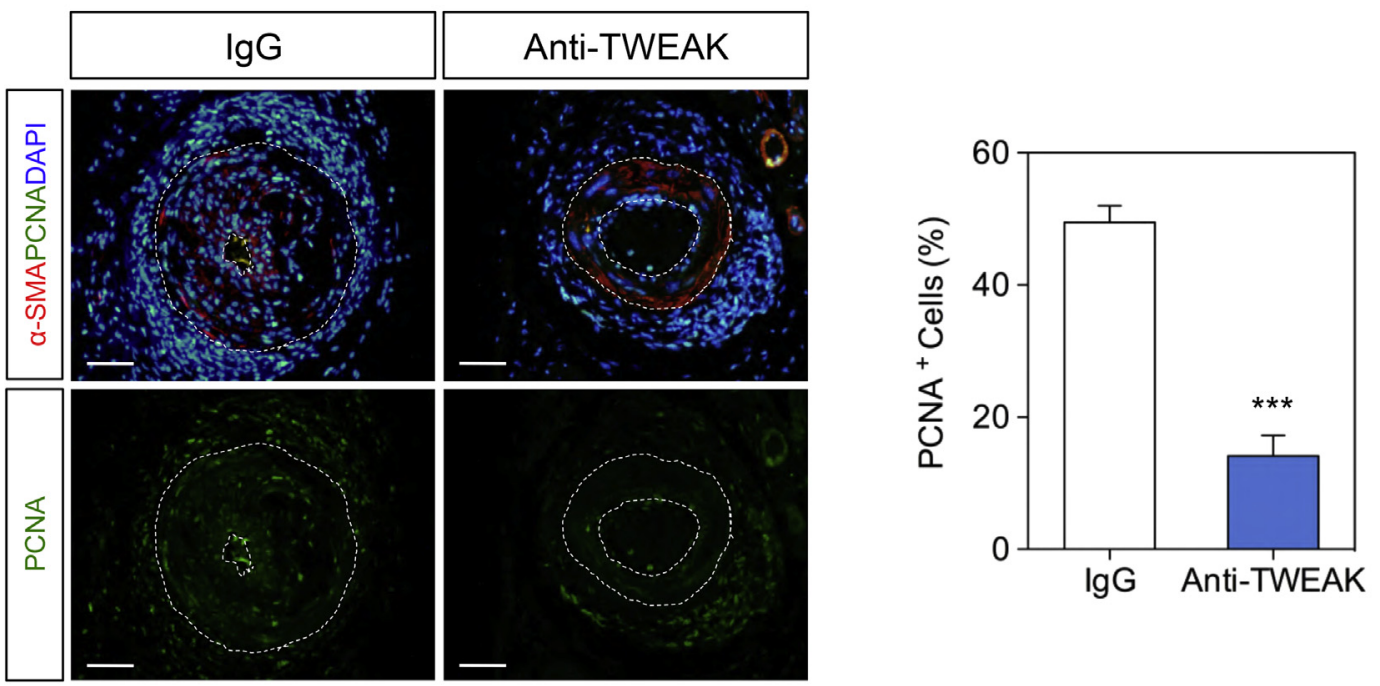

e
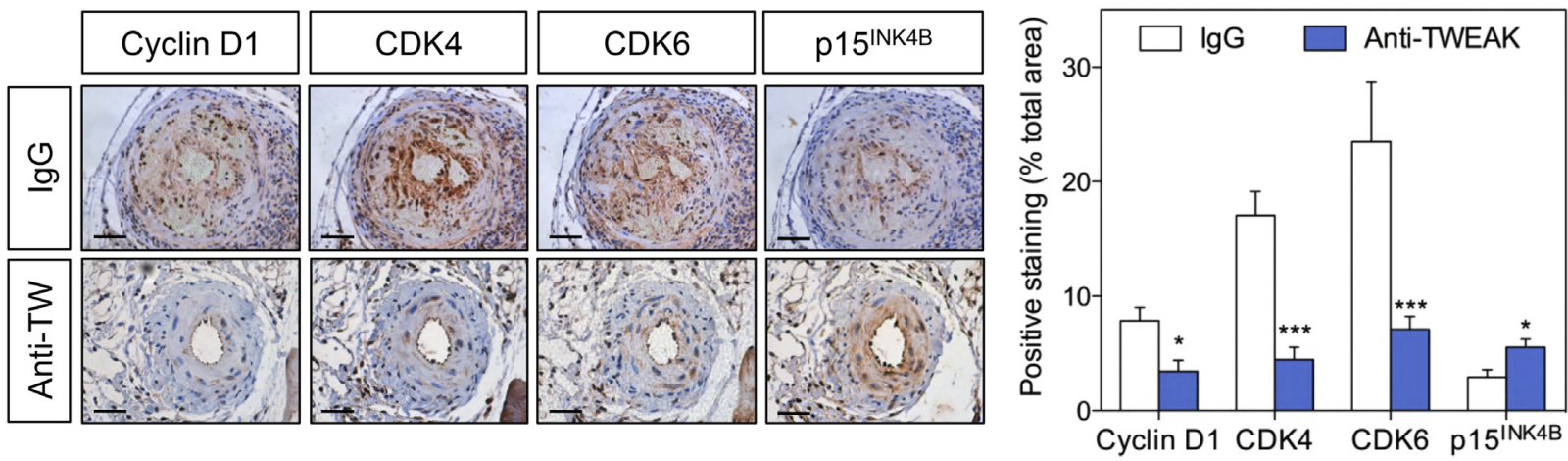
upregulation induced by TWEAK (Fig. 4E). No effect was observed with NF-kB inhibitor parthenolide (Fig. 4E).

\subsection{Loss of Tnfrsf12a or Tnfsf12 decreases neointimal formation in femoral artery after wire injury}

VSMCs migration from media to intima and their subsequent proliferation is a pathological manifestation of restenosis after angioplasty [29]. To corroborate the in vitro findings, we next tested the functional role of the TWEAK/Fn14 axis in VSMCs-rich lesion formation in vivo performing a guidewire injury in femoral arteries of mice. This experimental model that mimics the damage caused by angioplasty in humans [30]. The gene and protein expression levels of TWEAK and Fn14 were increased in femoral artery after 14 days of wire injury in WT mice compared with non-injured arteries (Fig. 5A-B). Immunohistochemistry analysis also showed a predominant localization of both TWEAK and Fn14 protein in the medial and neointimal layers (Fig. 5B). The majority of cells present in the neointima were positive for markers of VSMCs such as $\alpha$-SMA and calponin, and also for TWEAK and Fn14 (Fig. S4). These data should indicate that the in vivo phenotype observed in our animal model is mainly dependent of VSMC activity. We next performed a guide-wire injury in femoral arteries of Tnfrsf12a and Tnfsf12 deficient mice to analyze the role of TWEAK and Fn14 in vascular remodeling. Wire injury-mediated neointimal formation determined by intima area and intima to media ratio was significantly smaller in Tnfrsf $12 a^{-/-}$and Tnfs $f 12^{-/-}$compared with WT mice (Fig. 5C-D). In addition, the number of cells per $\mathrm{mm}^{2}$ was also decreased in the femoral sections of Tnfrsf $12 a^{-/-}$or Tnfsf $12^{-/-}$compared with WT mice (Fig. 5D).

Proliferating cell nuclear antigen (PCNA) positive cells in wire-injured femoral arteries were significantly reduced in either Tnfrsf $12 a^{-/-}$or Tnfsf $12^{-1-}$ femoral cross-sections compared with WT mice (Fig. 6A). We next tested how the TWEAK or Fn14 absence impacts on the expression of cell cycle regulators. We found that lack of Tnfrsf12a or Tnfsf12 increased Cdkn2B and decreased Cdk4, Cdk6 and CcnD1 mRNA expression in injured femoral arteries with respect to WT mice (Fig. 6B). Consistently, cyclin D1, CDK4 and CDK6 protein levels were markedly reduced and $\mathrm{p} 15^{\mathrm{INK} 4 \mathrm{~B}}$ increased in the injured femoral arteries of Tnfrsf12a or Tnfsf12 deficient mice compared with WT mice (Fig. 6C). These results indicate that TWEAK/Fn14 axis play a key role in neointimal formation through the regulation of the cell cycle by reduction of $\mathrm{p} 15^{\mathrm{INK} 4 \mathrm{~B}}$ expression and increase of CDK4/CDK6 and cyclin D1 expression.

\subsection{Treatment with anti-TWEAK antibody reduces neointimal formation after wire injury in mice}

To analyze the protective effect of TWEAK-based therapy against injury-induced neointimal hyperplasia, WT mice were treated with anti-TWEAK mAb or control IgG (10 mg/kg/twice a week). Mice were treated the day before the wire injury was performed and during 14 days after this procedure (Fig. 7A). Neointimal formation determined by intima area and intima to media ratio was significantly diminished in anti-TWEAK-treated compared to IgG-treated mice (Fig. 7B-C). In addition, the number of cells $/ \mathrm{mm}^{2}$ was also reduced in femoral sections from anti-TWEAK treated mice compared with IgG treated mice (Fig. 7B-C). Consistent with data obtained from Tnfsf $12^{-1-}$ mice, femoral cross-sections from anti-TWEAK treated mice showed a 71\% reduction in $\mathrm{PCNA}^{+}$cells compared with IgG-treated mice (Fig. 7D). Finally, we observed reduced protein expression of cyclin D1 and CDK4/CDK6 alongside with increased $\mathrm{p} 15^{\mathrm{INK} 4 \mathrm{~B}}$ levels in injured femoral arteries of mice treated with anti-TWEAK compared with those treated with IgG (Fig. 7E). Collectively, these data provide evidence that anti-TWEAK $\mathrm{mAb}$ treatment ameliorates restenosis after angioplasty.

\subsection{TWEAK/Fn14 axis in human intimal thickening}

Finally, in order to translate the results obtained from our in vivo model to the human context, we have performed $\alpha$-SMA, calponin, Fn14, TWEAK and PCNA immunohistochemistry in human healthy and stenotic coronary arteries. TWEAK was expressed in healthy coronary arteries while Fn14 expression was absent. In addition, we observed that both, TWEAK and Fn14 are expressed in human coronary arteries with stenosis (Fig. 8) as well as in-stent restenosis (Fig. 8) colocalizing with markers of VSMCs such as $\alpha$-SMA and calponin. $\mathrm{PCNA}^{+}$cells are also present in the neointima of both types of human injured coronary arteries, but not in healthy artery (Fig. 8).

\section{Discussion}

Percutaneous transluminal coronary angioplasty has been widely used to open up blocked coronary arteries [6]. However, many patients undergoing coronary angioplasty experience postangioplasty restenosis, which is a major obstacle in the long-term outcome of angioplasty interventions. Restenosis is defined as the healing response of the arterial wall to mechanical injury and implicates neointimal hyperplasia (VSMCs proliferation and migration) and vessel remodeling. It has been previously demonstrated that TWEAK participates in several pathologies that course with vascular remodeling. In this sense, TWEAK increases the inflammatory response associated with atherosclerotic plaque development in mice $[13,14]$. In addition, TWEAK and Fn14 participate in matrix degradation and induce angiogenesis in experimental abdominal aortic aneurysm [10]. Although it is known that VSMCs are key cells implicated in vascular remodeling, there are so far no studies focusing on the TWEAK-regulated signaling pathways in VSMCs. Here, we provide the first evidence for a pivotal role of TWEAK/Fn14 axis in neointimal formation after angioplasty. We used RNA-Seq to systematically investigate the global transcriptome of cultured VSMCs incubated in the presence or absence of TWEAK. In this manner we generated a useful resource for understanding the effect of TWEAK/Fn14 interaction on VSMCs biology. RNA-Seq and network analyses unveiled that one of the main actions of TWEAK in VSMCs is cell proliferation regulation. Our in vivo findings indicate that: i) both TWEAK and Fn14 mRNA and protein expression are induced in vascular wall after injury in wild type mice; ii) gene deletion of TWEAK and Fn14 in Tnfsf12 or Tnfrsf12a deficient mice protects from cell proliferation and neointimal formation; iii) anti-TWEAK treatment prevents cell proliferation and neointimal formation in injured arteries; iv) TWEAK and Fn14 are expressed in human coronary in-stent restenosis. Furthermore, our mechanistic studies demonstrate that TWEAK interaction with its cognate receptor Fn14 induces the proliferation and migration of VSMCs through the concomitant activation of CDK4/6 and cyclin D1 and inhibition of p15 $5^{\text {INK4B }}$ expression. Moreover, p15 $5^{\mathrm{INK} 4 \mathrm{~B}}$ and cyclin D1 expression are regulated by ERK1/2 and Akt kinases. Therefore, this work defines a hitherto unknown role of TWEAK/Fn14 axis during the development of restenosis after angioplasty (Fig. 9).

In vitro experiments in VSMCs confirmed that TWEAK, through its receptor Fn14, promotes VSMCs proliferation. The proliferative action

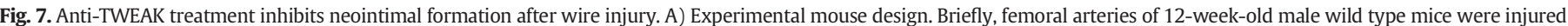

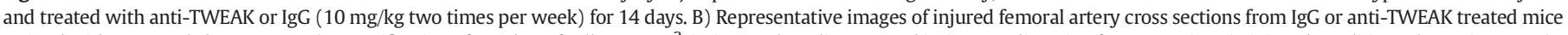

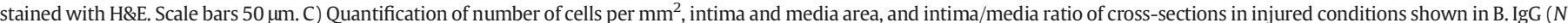

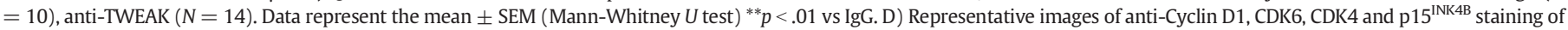

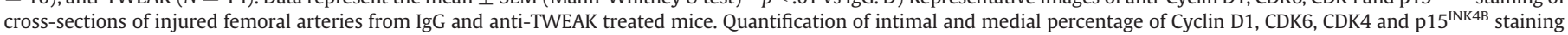
respectively, in the injured femoral artery of $\operatorname{IgG}(\mathrm{N}=10)$, anti-TWEAK $(N=11)$. Data represent the mean $\pm \mathrm{SEM}(\mathrm{Mann}-\mathrm{Whitney} \mathrm{U}$ test $){ }^{* *} p<.01 \mathrm{vs} \operatorname{IgG}$. 

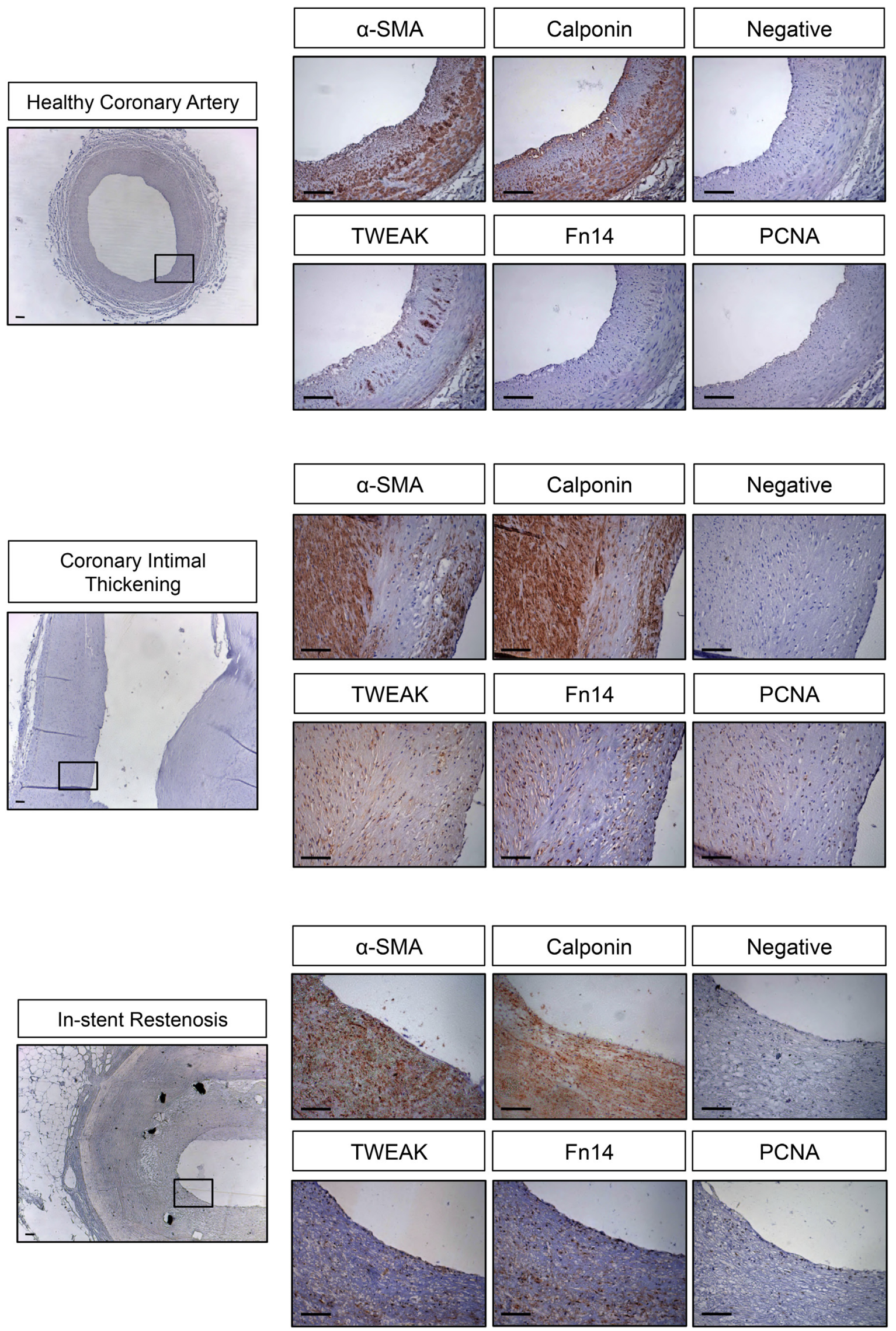

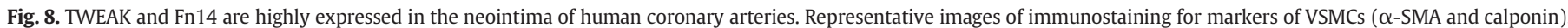
TWEAK, Fn14 or PCNA in serial section from human coronary artery with or without stenosis. Negative controls were stained with non-specific IgG. Scale bars 100 um. 


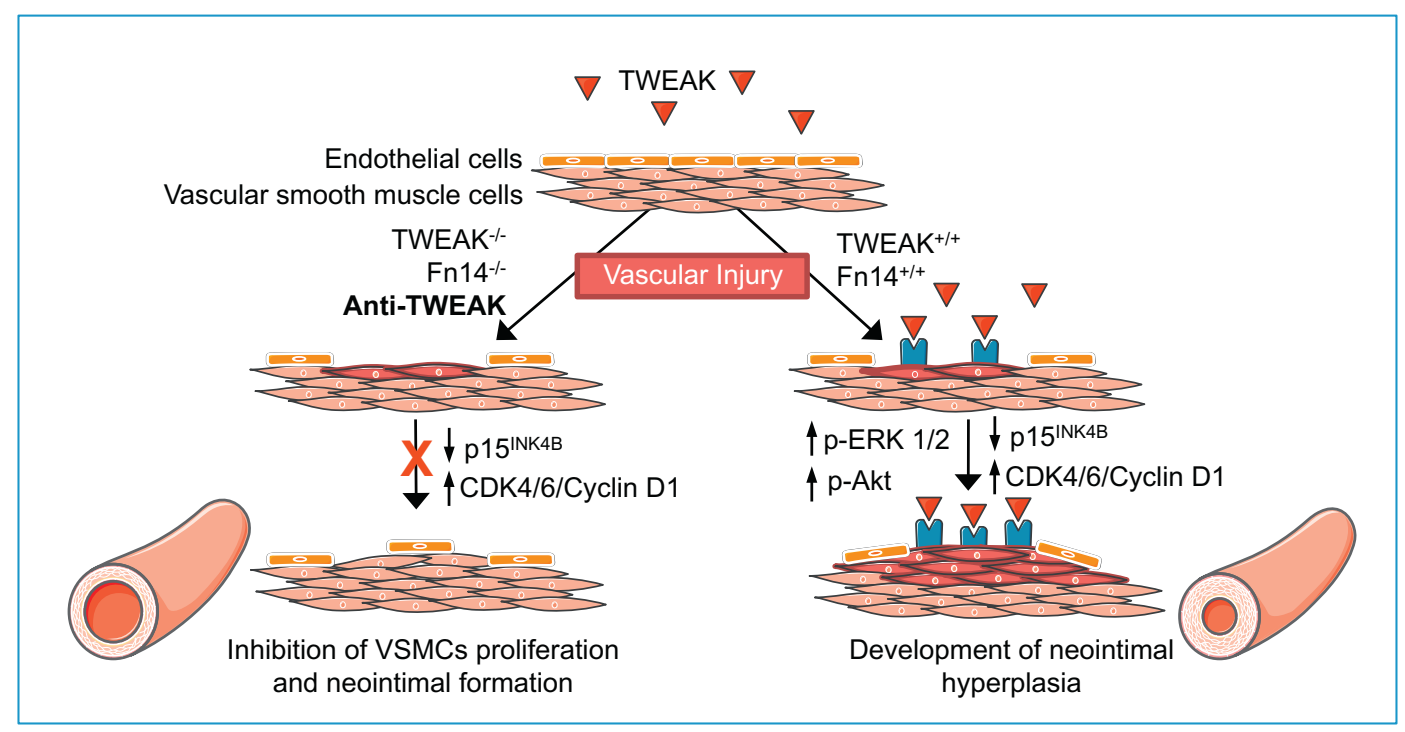

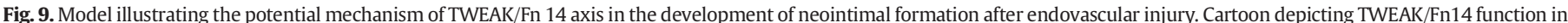

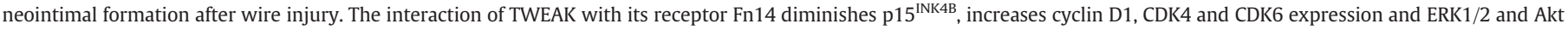
activation in VSMCs, leading to an increase in VSMCs proliferation and migration. Therapeutic intervention with anti-TWEAK antibodies reduces neointimal formation.

of TWEAK has been previously reported in several cell types including endothelial cells, cardiomyocytes, cardiac fibroblast, keratinocytes, hepatic cells and several tumoral cell lines [7,31-33]. Although the mechanisms by which TWEAK induces cell proliferation are poorly known, it has been previously shown that TWEAK induces cyclin D2 upregulation and $227^{\text {kip } 1}$ downregulation in cardiomyocytes, effect dependent of ERK and PI3K signaling [32]. Now, we demonstrate for the first time that TWEAK decreases the expression of $\mathrm{p} 15^{\mathrm{INK} 4 \mathrm{~B}}$, a cyclin-dependent kinase inhibitor implicated in cell cycle regulation. TWEAK-mediated p15 $15^{\text {INK4B }}$ downregulation was related to the interaction with its sole receptor Fn14. Thus, VSMCs from Tnfrsf12a $a^{-1-}$ mice or siRNA against Fn14 prevented the downregulation of $\mathrm{p} 15^{\mathrm{INK} 4 \mathrm{~B}}$ induced by TWEAK.

The $\mathrm{p} 15^{\mathrm{INK} 4 \mathrm{~B}}$ protein participates in the maintenance of the quiescent state in different cells. In VSMCs, $\mathrm{p} 15^{\mathrm{INK} 4 \mathrm{~B}}$ upregulation results in G1 phase arrest and inhibition of pRb phosphorylation [34]. In addition, overexpression of $\mathrm{p} 15^{\mathrm{INK} 4 \mathrm{~B}}$ in vivo inhibits in-stent intimal hyperplasia in rabbits [34]. However, although genetic deletion of $C d k n 2 B$ in mice accelerates VSMCs proliferation, paradoxically it leads to smaller neointimal lesions [35]. Now, we observed a downregulation of $\mathrm{p} 15^{\mathrm{INK} 4 \mathrm{~B}}$ expression in TWEAK-treated VSMC and, an increase of $\mathrm{p} 15^{\mathrm{INK} 4 \mathrm{~B}}$ levels in Tnfrsf12 $a$ and Tnfsf12 deficient mice, an effect associated with a decrease in cell proliferation. Based on these results, it is conceivable to think that the effect of $\mathrm{p} 15^{\mathrm{INK} 4 \mathrm{~B}}$ in neointimal formation is dependent on its expression level within the arterial wall. The precise mechanism by which TWEAK decreases $\mathrm{p} 15^{\mathrm{INK} 4 \mathrm{~B}}$ should be related with the activation of different pathways. In endothelial cells, TWEAK activates ERK1/2, JNK $1 / 2$ and NF-kB, but not p38 MAPK [31]. In addition, TWEAK activates PI3K/Akt in osteoblastic cells [36] and ERK1/2 in human VSMCs [37]. Now, we observed that TWEAK induces ERK1/2, Akt and p65 phosphorylation in VSMCs. Accordingly, ERK activation seems to be essential for cell cycle progression in VSMCs [38]. Using specific inhibitors, we observed that inhibition of ERK1/2 and Akt activation prevented p15 $5^{\text {INK4B }}$ downregulation, indicating that the mechanism by which TWEAK decreases $15^{\mathrm{INK} 4 \mathrm{~B}}$ expression it may be related to ERK1/2 and Akt activation. We have also observed that TWEAK induces a rapid p65, Akt and ERK phosphorylation, followed by a secondary and delayed phosphorylation of these proteins. The mechanisms ruling the bimodal activation of p65, Akt and ERK are unknown. This could be due by to the fact that TWEAK upregulates several molecules such as cytokines, chemokines and other members of the TNF superfamily that could be responsible for the secondary signal observed. p15 ${ }^{\text {INK4B }}$ is a known inhibitor of CDK4/CDK6 and cyclin D1 expression in VSMCs [28]. Accordingly, we observed an increase in CDK4/ CDK6 and cyclin D1 mRNA and protein expression under TWEAK stimulation in VSMCs. The increase of these cyclin-dependent kinases and cyclin D1 was accompanied by an augmentation in the number of cells in S phase and total number of cells, and a decrease of cells in G0/G1. Very importantly, we also provide evidence that our in vitro observations are applicable in vivo by demonstrating that Tnfrsf $12 a$ or Tnfsf12 deletion decreased the number of proliferative cells, CDK4/6 and cyclin D1 expression, and increased $\mathrm{p} 15^{\mathrm{INK} 4 \mathrm{~B}}$ expression, ending in less neointimal formation in a murine model of vascular injury.

VSMCs proliferation is associated with their plasticity [39]. VSMCs have the ability to change from a differentiated and quiescent contractile state to a proliferative and migratory synthetic phenotype in response to surrounding stimulus. In this sense, we have previously demonstrated that TWEAK-stimulated VSMCs differentiate from a contractile to a synthetic phenotype [15]. Thus, TWEAK decreases markers of contractile phenotype such as $\alpha$-SMA and calponin, and increases markers of synthetic phenotype such as osteopontin and metalloproteinase 9 in cultured VSMCs $[10,15]$. Change in the phenotype induced by TWEAK is in agreement with the increase in VSMCs proliferation observed in our study. However, it is important to note that many expanded VSMC-derived cells in the injured-induced neointima maintain $\alpha$-SMA expression [40]. In this context, it has been previously demonstrated that TWEAK/Fn14 axis increases $\alpha$-SMA expression during myofibroblasts differentiation [41]. VSMCs migration is also an important component that contributes to neointimal formation [4]. Although it has been reported that TWEAK increases cell migration in a variety of cell types such as endothelial cells [42], data regarding the effect of TWEAK in VSMCs migration were lacking. Here we demonstrate that TWEAK induces VSMCs motility. This effect is directly related with the expression of Fn14 since cells lacking Tnfrsf12a gene failed to migrate in response to TWEAK. Therefore, the induction of cell migration by TWEAK/Fn14 axis can also contribute to increase lesion formation in vivo.

The most important finding in our study is the potential translationality of our results obtained from the use of anti-TWEAK therapy to limit neointimal formation after wire injury. Although the introduction of drug-eluting stents has improved the prevention and treatment of restenosis [5], neointimal obstruction persists in most cases [43]. In addition, the cytostatic agents such as rapamycin are 
relatively nonspecific and are associated with late-stent thrombosis [5]. Our finding demonstrated that blockade of TWEAK by specific antibodies can recapitulate the genetic phenotype obtained in Tnfsf $12^{-1-}$ mice preventing cell proliferation after injury by increasing $\mathrm{p} 15^{\mathrm{INK} 4 \mathrm{~B}}$ expression and reducing CDK4/6 and cyclin D1 expression levels in injured vessel. However, it is important to note that VSMCs proliferation could impact other vascular remodeling processes such as atherosclerosis. In fact, while VSMCs proliferation is detrimental in the early stages of atherosclerotic plaque developing, it is protective in advanced atherosclerotic lesions, preventing fibrous cap from rupturing and promoting plaque repair [44]. For that reason, a local administration of TWEAK/ Fn14 blockers through drug-eluting stents could be a better option to lower the restenosis rate.

In conclusion, our findings provide novel and important insights into the role of TWEAK/Fn14 axis in neointimal formation after wire injury. TWEAK increases VSMCs proliferation and migration through $\mathrm{p} 15^{\mathrm{INK} 4 \mathrm{~B}}$ downregulation and CDK4/6 and cyclin D1 upregulation. Therapies aimed to block TWEAK/Fn14 interaction could protect from restenosis after angioplasty.

Supplementary data to this article can be found online at https://doi. org/10.1016/j.ebiom.2019.07.072.

\section{Funding sources}

This work was supported by Instituto de Salud Carlos III (Fondo de Investigaciones Sanitarias ISCiii/FEDER PI13/00395; PI16/01419; PI17/ 01495) and Spanish Biomedical Research Centre in Cardiovascular Disease (CIBERCV) and Metabolic Diseases and Diabetes (CIBERDEM). PM was supported by ISCIII Miguel Servet Program (CP16/00116). CGM was supported by Fundación Conchita Rábago. NMB and VE were supported by the Spanish Ministry of Economy and Competitiveness (Juan de la Cierva IJCI-2016-29630 and Ramón y Ramón Cajal Program RyC-2013-12880, respectively). JMM has been supported a postdoctoral fellowship from the American Diabetes Association (Grant 1-15-MI-03) and a postdoctoral fellowship from the American Heart Association. The funders had no role in study design, data collection and analysis, decision to publish, or preparation of the manuscript.

\section{Author contributions statement}

The study was conceived by JE, JLMV and LMBC. NMB and LMBC designed the study and analyzed the data. NMB, CGM and VE performed the experiments. JMM and PM performed RNA-Seq and analyzed the obtained data. JBM performed human samples studies. NMB and LMBC wrote the manuscript with contributions of JLMV and VE. All authors read and approved the manuscript.

\section{Declaration of Competing Interest}

None.

\section{Acknowledgments}

We thank to Dr. Linda Burkly (Biogen, Inc. Boston, MA, USA) for contributing to the conception and design of the study, Dr. Carmen GómezGuerrero (IIS-FJD, Madrid, Spain) for critical suggestions, Dr. Jose Martínez-Gonzalez (IIBB-CSIC Sant Pau, Barcelona, Spain) for providing healthy human coronary arteries and Patricia Quesada (IIS-FJD, Madrid, Spain) for her technical assistance.

\section{References}

[1] Dzau VJ, Braun-Dullaeus RC, Sedding DG. Vascular proliferation and atherosclerosis: new perspectives and therapeutic strategies. Nat Med 2002;8:1249-56.

[2] Alexander MR, Owens GK. Epigenetic control of smooth muscle cell differentiation and phenotypic switching in vascular development and disease. Annu Rev Physiol 2012;74:13-40.
[3] Libby P, Hansson GK. Inflammation and immunity in diseases of the arterial tree: players and layers. Circ Res 2015;116:307-11.

[4] Chaabane C, Otsuka F, Virmani R, Bochaton-Piallat M-L. Biological responses in stented arteries. Cardiovasc Res 2013;99:353-63.

[5] Iakovou I, Schmidt T, Bonizzoni E, Ge L, Sangiorgi GM, Stankovic G, et al. Incidence predictors, and outcome of thrombosis after successful implantation of drugeluting stents. JAMA 2005;293:2126-30.

[6] Jukema JW, Ahmed TAN, Verschuren JJW, Ouax PHA. Restenosis after PCI. Part 2: prevention and therapy. Nat Rev Cardiol 2011;9:79-90.

[7] Winkles JA. The TWEAK-Fn14 cytokine-receptor axis: discovery, biology and therapeutic targeting. Nat Rev Drug Discov 2008;7:411-25.

[8] Burkly LC, Michaelson JS, Zheng TS. TWEAK/Fn14 pathway: an immunological switch for shaping tissue responses. Immunol Rev 2011;244:99-114.

[9] Munoz-Garcia B, Martin-Ventura JL, Martínez E, Sánchez S, Hernández G, Ortega L, et al. Fn14 is upregulated in cytokine-stimulated vascular smooth muscle cells and is expressed in human carotid atherosclerotic plaques: modulation by atorvastatin. Stroke 2006;37:2044-53.

[10] Tarín C, Fernández-Laso V, Sastre C, Madrigal-Matute J, Gómez M, Zaragoza C, et al Tumor necrosis factor-like weak inducer of apoptosis or Fn14 deficiency reduce elastase perfusion-induced aortic abdominal aneurysm in mice. J Am Heart Assoc 2014; 3 (pii:e000723).

[11] Sastre C, Fernández-Laso V, Madrigal-Matute J, Munoz-Garcia B, Moreno JA, PastorVargas C, et al. Genetic deletion or TWEAK blocking antibody administration reduce atherosclerosis and enhance plaque stability in mice. J Cell Mol Med 2014;18: 721-34.

[12] Blanco-Colio LM. TWEAK/Fn14 axis: a promising target for the treatment of cardiovascular diseases. Front Immunol 2014;5:3.

[13] Munoz-Garcia B, Moreno JA, Lopez-Franco O, Sanz AB, Martin-Ventura JL, Blanco J, et al. Tumor necrosis factor-like weak inducer of apoptosis (TWEAK) enhances vascular and renal damage induced by hyperlipidemic diet in ApoE-knockout mice. Arterioscler Thromb Vasc Biol 2009;29:2061-8.

[14] Schapira K, Burkly LC, Zheng TS, Wu P, Groeneweg M, Rousch M, et al. Fn14-fc fusion protein regulates atherosclerosis in ApoE-/- mice and inhibits macrophage lipid uptake in vitro. Arterioscler Thromb Vasc Biol 2009;29:2021-7.

[15] Fernández-Laso V, Sastre C, Méndez-Barbero N, Egido J, Martín-Ventura JL, GómezGuerrero C, et al. TWEAK blockade decreases atherosclerotic lesion size and progression through suppression of STAT1 signaling in diabetic mice. Sci Rep 2017;7:46679.

[16] Montaner D, Dopazo J. Multidimensional gene set analysis of genomic data. PLoS One 2010;5:e10348.

[17] Alonso R, Salavert F, Garcia-Garcia F, Carbonell-Caballero J, Bleda M, Garcia-Alonso L, et al. Babelomics 5.0: functional interpretation for new generations of genomic data. Nucleic Acids Res 2015;43(W1):W117-21.

[18] Garcia-Alonso L, Alonso R, Vidal E, Amadoz A, de María A, Minguez P, et al. Discovering the hidden sub-network component in a ranked list of genes or proteins derived from genomic experiments. Nucleic Acids Res 2012;40:e158.

[19] Al-Shahrour F, Minguez P, Tarraga J, Medina I, Alloza E, Montaner D, et al. FatiGO +: a functional profiling tool for genomic data. Integration of functional annotation, regulatory motifs and interaction data with microarray experiments. Nucleic Acids Res 2007;35:W91-6.

[20] Wu CH, Pan JS, Chang WC, Hung JS, Mao SJT. The molecular mechanism of actinomycin $\mathrm{D}$ in preventing neointimal formation in rat carotid arteries after balloon injury. J Biomed Sci 2005;12:503-12.

[21] Terzian Z, Gasser TC, Blackwell F, Hyafil F, Louedec L, Deschildre C, et al. Peristrut microhemorrhages: a possible cause of in-stent neoatherosclerosis? Cardiovasc Pathol 2017;26:30-8.

[22] Franck G, Mawson TL, Folco EJ, Molinaro R, Ruvkun V, Engelbertsen D, et al. Roles of PAD4 and NETosis in experimental atherosclerosis and arterial injury: implications for superficial erosion. Circ Res 2018;123:33-42.

[23] Franck G, Even G, Gautier A, Salinas M, Loste A, Procopio E, et al. Haemodynamic stress-induced breaches of the arterial intima trigger inflammation and drive atherogenesis. Eur Heart J 2019;40:928-37.

[24] Girgenrath M, Weng S, Kostek CA, Browning B, Wang M, Brown SAN, et al. TWEAK, via its receptor Fn14, is a novel regulator of mesenchymal progenitor cells and skeletal muscle regeneration. EMBO J 2006;25:5826-39.

[25] Dohi T, Borodovsky A, Wu P, Shearstone JR, Kawashima R, Runkel L, et al. TWEAK/ Fn14 pathway: a nonredundant role in intestinal damage in mice through a TWEAK/intestinal epithelial cell axis. Gastroenterology 2009;136:912-23.

[26] Esteban V, Méndez-Barbero N, Jiménez-Borreguero LJ, Roqué M, Novensá L, GarcíaRedondo $\mathrm{AB}$, et al. Regulator of calcineurin 1 mediates pathological vascular wall remodeling. J Exp Med 2011;208:2125-39.

[27] Sherr C], Roberts JM. Living with or without cyclins and cyclin-dependent kinases. Genes Dev 2004;18:2699-711.

[28] Mathew OP, Ranganna K, Yatsu FM. Butyrate, an HDAC inhibitor, stimulates interplay between different posttranslational modifications of histone $\mathrm{H} 3$ and differently alters G1-specific cell cycle proteins in vascular smooth muscle cells. Biomed Pharmacother 2010;64:733-40.

[29] Berk BC. Vascular smooth muscle growth: autocrine growth mechanisms. Physiol Rev 2001;81:999-1030.

[30] Roque M, Fallon JT, Badimon JJ, Zhang WX, Taubman MB, Reis ED. Mouse model of femoral artery denudation injury associated with the rapid accumulation of adhesion molecules on the luminal surface and recruitment of neutrophils. Arterioscler Thromb Vasc Biol 2000;20:335-42.

[31] Donohue PJ, Richards CM, Brown SAN, Hanscom HN, Buschman J, Thangada S, et al TWEAK is an endothelial cell growth and chemotactic factor that also potentiates FGF-2 and VEGF-A mitogenic activity. Arterioscler Thromb Vasc Biol 2003;23: 594-600. 
[32] Novoyatleva T, Diehl F, van Amerongen MJ, Patra C, Ferrazzi F, Bellazzi R, et al. TWEAK is a positive regulator of cardiomyocyte proliferation. Cardiovasc Res 2010;85:681-90.

[33] Gao H-X, Campbell SR, Burkly LC, Jakubowski A, Jarchum I, Banas B, et al. TNF-like weak inducer of apoptosis (TWEAK) induces inflammatory and proliferative effects in human kidney cells. Cytokine 2009;46:24-35.

[34] Segev A, Nili N, Qiang B, Osherov AB, Giordano FJ, Jaffe R, et al. Inhibition of intimal hyperplasia after stenting by over-expression of p15: a member of the INK4 family of cyclin-dependent kinase inhibitors. J Mol Cell Cardiol 2011;50:417-25.

[35] Leeper NJ, Raiesdana A, Kojima Y, Kundu RK, Cheng H, Maegdefessel L, et al. Loss of CDKN2B promotes p53-dependent smooth muscle cell apoptosis and aneurysm formation. Arterioscler Thromb Vasc Biol 2013;33:e1-10.

[36] Ando T, Ichikawa J, Wako M, Hatsushika K, Watanabe Y, Sakuma M, et al. TWEAK/ Fn14 interaction regulates RANTES production, BMP-2-induced differentiation, and RANKL expression in mouse osteoblastic MC3T3-E1 cells. Arthritis Res Ther 2006; 8:R146.

[37] Hénaut L, Sanz AB, Martin-Sanchez D, Carrasco S, Villa-Bellosta R, AldamizEchevarria G, et al. TWEAK favors phosphate-induced calcification of vascular smooth muscle cells through canonical and non-canonical activation of NFkB. Cell Death Dis 2016;7:e2305.

[38] Sedding DG, Tröbs M, Reich F, Walker G, Fink L, Haberbosch W, et al. 3Deazaadenosine prevents smooth muscle cell proliferation and neointima formation by interfering with Ras signaling. Circ Res 2009;104:1192-200.
[39] Yoshida T, Owens GK. Molecular determinants of vascular smooth muscle cell diversity. Circ Res 2005;96:280-91.

[40] Chappell J, Harman JL, Narasimhan VM, Yu H, Foote K, Simons BD, et al. Extensive proliferation of a subset of differentiated, yet plastic, medial vascular smooth muscle cells contributes to neointimal formation in mouse injury and atherosclerosis models. Circ Res 2016;119:1313-23.

[41] Novoyatleva T, Schymura Y, Janssen W, Strobl F, Swiercz JM, Patra C, et al. Deletion of Fn14 receptor protects from right heart fibrosis and dysfunction. Basic Res Cardiol 2013;108:325.

[42] Wiley SR, Cassiano L, Lofton T, Davis-Smith T, Winkles JA, Lindner V, et al. A novel TNF receptor family member binds TWEAK and is implicated in angiogenesis. Immunity 2001;15:837-46

[43] Waseda K, Miyazawa A, Ako J, Hasegawa T, Tsujino I, Sakurai R, et al. Intravascular ultrasound results from the ENDEAVOR IV trial: randomized comparison between zotarolimus- and paclitaxel-eluting stents in patients with coronary artery disease. JACC Cardiovasc Interv 2009;2:779-84.

[44] Bennett MR, Sinha S, Owens GK. Vascular smooth muscle cells in atherosclerosis. Circ Res 2016:118:692-702. 